TRANSACTIONS OF THE

AMERICAN MATHEMATICAL SOCIETY

Volume 354, Number 6, Pages 2131-2171

$\mathrm{S} 0002-9947(02) 02963-\mathrm{X}$

Article electronically published on February 14, 2002

\title{
SUBSPACES OF NON-COMMUTATIVE SPACES
}

\author{
S. PAUL SMITH
}

\begin{abstract}
This paper concerns the closed points, closed subspaces, open subspaces, weakly closed and weakly open subspaces, and effective divisors, on a non-commutative space.
\end{abstract}

\section{INTRODUCTION}

This paper examines non-commutative analogues of some of the elementary ideas of algebraic geometry. Our wish is to have a language for non-commutative algebraic geometry that is geometric and topological. This will, eventually, allow us to use our geometric intuition in situations that at present are viewed almost exclusively from an algebraic perspective. One can already see hints of this goal in Gabriel's thesis 6]. More recently, Rosenberg and Van den Bergh have given new life to this program. We further extend their ideas by examining the interactions between the following basic objects: weakly closed and weakly open subspaces, closed subspaces, open subspaces, closed points, and effective divisors.

We follow Rosenberg [20] 21] and Van den Bergh [25] in taking a Grothendieck category as our basic non-commutative geometric object. Thus a non-commutative space $X$ is a Grothendieck category $\operatorname{Mod} X$. The idea for the notation $X=\operatorname{Mod} X$ is Van den Bergh's. We also use his notion of $X$ - $X$-bimodules, and denote the unit object in the monoidal category of $X$-X-bimodules by $o_{X}$. The bifunctors $\mathcal{E} x t_{X}^{i}$ and $\mathcal{T}_{\text {or }}^{X}$ X are defined in 25.

The standard commutative example of a space is the category of quasi-coherent sheaves on a quasi-separated, quasi-compact scheme. We will speak of a quasiseparated, quasi-compact scheme $X$ as a space with the understanding that Mod $X$ is $Q \operatorname{coh} X$. The two non-commutative models are $\operatorname{Mod} R$, the category of right modules over a ring, and $\operatorname{Proj} A$, the non-commutative projective spaces having a (not necessarily commutative) graded ring $A$ as homogeneous coordinate ring (Definition 2.2).

The notion of a weakly closed subspace of a non-commutative space first appeared in Gabriel's thesis [6, page 395] - he calls a full subcategory of an abelian category closed if it is closed under subquotients and direct limits. We will modify his terminology, and say that a full subcategory of a Grothendieck category is weakly closed if it satisfies these conditions. Closure under subquotients ensures that the inclusion functor is exact, and closure under direct limits ensures that the

Received by the editors November 28, 2000 and, in revised form, April 5, 2001 and September 20, 2001.

2000 Mathematics Subject Classification. Primary 14A22; Secondary 18E15, 16S38, 16P40, 18 F99.

The author was supported by NSF grant DMS-0070560.

(C)2002 American Mathematical Society 
inclusion functor has a right adjoint. If the inclusion functor also has a left adjoint we say that the subcategory is closed. This modification of Gabriel's terminology makes it compatible with the language of algebraic geometry: the closed subcategories (in our sense) of $\operatorname{Mod} R$ are in bijection with the two-sided ideals of $R$; and, every closed subscheme of a scheme $X$ determines a closed subspace of $\mathrm{Q} c \mathrm{~h} X$. If $X$ is a quasi-projective scheme over a field, then the closed subspaces are the same things as the closed subschemes (Theorem 4.1). Our terminology is also compatible with Van den Bergh's notion of weak ideals; the weakly closed subspaces of a space $X$ are in bijection with the weak ideals in $o_{X}$, the identity functor on $\operatorname{Mod} X$.

We define the complement to a weakly closed subspace in the obvious way (Definition 6.4), and define weakly open subspaces (Definition 2.5) in such a way that they are precisely the complements to the weakly closed subspaces when the ambient space is locally noetherian. The intersection of an arbitrary collection, and the union of a finite collection, of weakly closed subspaces (Definitions 3.2 and 3.4) have already been defined by Rosenberg; these are again weakly closed subspaces. Because a weakly open subspace is a complement to a weakly closed subspace we can define the intersection (resp., union) of weakly open subspaces as the complement of the corresponding union (resp., intersection) of weakly closed subspaces. For the most part these ideas interact with each other in reasonable ways - for example, see Proposition 3.3, Lemmas 6.10, 6.16, 6.18, and 6.20, Corollary 7.4, and Propositions 7.1, 7.9, and 7.10, Furthermore, Proposition [7.5] shows that if $U$ and $V$ are weakly open subspaces of a locally noetherian space $X$, then $U \cap V$ is the fiber product $U \times_{X} V$ in the category of non-commutative spaces with maps being the morphisms. Despite these positive results we do not know whether $W \cap(Y \cup Z)$ is equal to $(W \cap Y) \cup(W \cap Z)$ when $W, Y$, and $Z$, are weakly closed subspaces; the analogous formula for weakly open subspaces is valid.

The notions of containment and intersection between a weakly closed subspace and a weakly open subspace (see Definitions 6.17, 7.2, and 7.8) do not behave as well as one might initially hope. An essential feature of non-commutative algebra is that there can be non-split extensions between non-isomorphic simple modules. As a result of this, it is possible for a non-commutative space $X$ to have weakly closed subspaces $W$ and $Z$ such that $W \cap Z=\phi$, but $Z$ is not contained in $X \backslash W$. This illustrates the limitations of the analogy with commutative algebraic geometry. More generally, $Z \backslash(W \cap Z)$ need not be contained in $X \backslash W$; Proposition 7.6 describes exactly when $Z \backslash(W \cap Z)$ is contained in $X \backslash W$.

We extend the notion of integrality to the non-commutative setting by defining prime subspaces and prime modules (see also 23]). Proposition4.4 shows that these new notions are compatible with the usual notion of integrality for a noetherian scheme having an ample line bundle. They are also compatible with the usual notion of a prime ideal in a non-commutative ring. Proposition 4.7 shows that for noetherian non-commutative spaces that are close to being commutative there is a bijection between what we call the prime subspaces of $X$ and the indecomposable injectives in $\operatorname{Mod} X$. This extends both Matlis' result for commutative notherian rings, and Gabriel and Cauchon's extension of Matlis' result to rings satisfying Gabriel's condition $(\mathrm{H})$ (fully bounded right noetherian rings).

In section 5 we define closed points of a space. A test case for our definition is the following. Let $A$ be a connected graded $k$-algebra and $\operatorname{Proj} A$ the non-commutative projective space having $A$ as a homogeneous coordinate ring. Artin-Tate-Van den Bergh [2] have defined the notion of a point module for $A$; the idea behind their 
definition is that point modules for $A$ should play the role of closed points on $\operatorname{Proj} A$. However, until now there has been no definition of "point" in non-commutative geometry. In section 5 we show that point modules over $A$ do yield closed points of $\operatorname{Proj} A$.

To motivate our definition of closed point, recall that a closed point $p$ on a scheme $X$ corresponds to a simple object $\mathcal{O}_{p}=\mathcal{O}_{X} / \mathfrak{m}_{p}$ in Qcoh $X$, where $\mathfrak{m}_{p}$ is the sheaf of ideals vanishing at $p$. This sets up a bijection between closed points of $X$ and simple objects in $Q \operatorname{coh} X$. Since the geometric objects in non-commutative geometry are Grothendieck categories, we define a closed point of a space $X$ to be a certain kind of full subcategory of $\operatorname{Mod} X$. That subcategory is required to be closed in the sense described above, and to consist of all direct sums of a simple $X$-module, say $\mathcal{O}_{p}$. The requirement that the subcategory be closed means that not all simple $X$-modules correspond to closed points. In particular, an infinite dimensional simple module over a finitely generated $k$-algebra does not correspond to a closed point; such simple modules behave like higher dimensional geometric objects (see the remarks after Proposition 5.8).

Sometimes even finite dimensional simple modules behave like higher dimensional geometric objects. The $\mathcal{D}$-module approach to the representation theory of semisimple Lie algebras, and the dimension of the characteristic variety, yield a dimension function that gives finite dimensional representations dimension greater than zero, thus reflecting the fact that such modules do not always behave like points. The behavior of the higher Ext-groups of finite dimensional simple modules also indicates that they behave like higher dimensional geometric objects.

A further example of this phenomenon occurs when $\alpha: \tilde{X} \rightarrow X$ is Van den Bergh's blowup for a non-commutative surface $X$. Sometimes $X$ can have a module of Krull dimension one whose strict transform is a simple $\tilde{X}$-module, say $S$. That simple module gives a closed point on $\tilde{X}$, but with regard to its intersection properties (defined in terms of Ext-groups) that simple $\tilde{X}$-module behaves more like a curve than a point (see [13]). This is reinforced by the fact that $\alpha_{*} S$ is the original module of Krull dimension one - in particular, $\alpha$ does not send this closed point of $\tilde{X}$ to a closed point of $X$. For many purposes it is better to think of $S$ as a geometric object of dimension one.

In Section 8 we examine effective divisors (as defined by Van den Bergh) on a non-commutative space. These are analogues of effective Cartier divisors; an effective divisor is defined in terms of an invertible ideal, say $o_{X}(-Y)$, in $o_{X}$. That ideal cuts out a closed subspace $Y$ on $X$. If $W$ is a weakly closed subspace of $X$, we give precise conditions under which $W \cap Y$ is an effective divisor on $W$. We also show that if $Y$ is ample, then $W \cap Y \neq \phi$ for all weakly closed subspaces $W$ of positive dimension. We give conditions under which the set of closed points in $X$ is the disjoint union of the sets of closed points in $Y$ and $X \backslash Y$. Effective divisors behave better than arbitrary closed (or weakly closed) subspaces with respect to intersection and containment of other subspaces.

Acknowledgements. We thank M. Artin, P. Jørgensen, S. Kovacs, M. Van den Bergh, and J. Zhang for helpful conversations. We thank C. Pappacena for asking questions that prompted Propositions 4.6 and 4.7 This work was begun while the author was visiting the University of Copenhagen during the summers of 1998 and 1999, and was continued during the workshop on Noncommutative Algebra at 
the Mathematical Sciences Research Institute at Berkeley in 2000. The author is grateful to both institutions for their hospitality and financial support.

\section{Preliminaries}

Throughout we work over a fixed commutative base ring $k$. All categories are $k$-linear and functors between them are $k$-linear.

Definition 2.1. A non-commutative space $X$ is a Grothendieck category $\operatorname{Mod} X$. Objects in $\operatorname{Mod} X$ are called $X$-modules. We say $X$ is locally noetherian if $\operatorname{Mod} X$ is locally noetherian (that is, if $\operatorname{Mod} X$ has a set of noetherian generators). If $X$ is locally noetherian, we write $\bmod X$ for the full subcategory of $\operatorname{Mod} X$ consisting of all noetherian $X$-modules.

The empty space $\phi$ is defined by declaring $\operatorname{Mod} \phi$ to be the zero category; that is, the abelian category having only one object and one morphism.

We write $\operatorname{Mod} R$ for the category of right modules over a ring $R$.

We say $X$ is affine if $\operatorname{Mod} X$ has a progenerator. A ring $R \operatorname{such}$ that $\operatorname{Mod} X$ is equivalent to $\operatorname{Mod} R$ is called a coordinate ring of $X$. It is easy to see that an affine space is locally noetherian if and only if one, and hence all, of its coordinate rings is right noetherian.

If $\left(X, \mathcal{O}_{X}\right)$ is a scheme, then the category $\operatorname{Mod} \mathcal{O}_{X}$ of all sheaves of $\mathcal{O}_{X}$-modules is a Grothendieck category. If $X$ is quasi-compact and quasi-separated (for example, if $X$ is a noetherian scheme), then the full subcategory of $\operatorname{Mod} \mathcal{O}_{X}$ consisting of the quasi-coherent $\mathcal{O}_{X}$-modules is a Grothendieck category [으, page 186]; we denote this category by $Q \operatorname{coh} X$. We will speak of a quasi-compact and quasi-separated scheme $X$ as a space with the tacit understanding that $\operatorname{Mod} X$ is $Q \operatorname{coh} X$. For example, Spec $\mathbb{Z}$ will denote the space whose module category is $\operatorname{Mod} \mathbb{Z}$.

The word "scheme" will be reserved for schemes in the usual sense of commutative algebraic geometry, and the word "space" will always mean a space in the sense of Definition 2.1

Non-commutative analogues of projective schemes were introduced in [3] and [27].

Definition 2.2. Let $k$ be a field. Let $A$ be an $\mathbb{N}$-graded $k$-algebra such that $\operatorname{dim}_{k} A_{n}<\infty$ for all $n$. Define $\operatorname{GrMod} A$ to be the category of $\mathbb{Z}$-graded $A$-modules with morphisms the $A$-module homomorphisms of degree zero. We write Fdim $A$ for the full subcategory of direct limits of finite dimensional modules. We define the quotient category

$$
\text { Tails } A=\operatorname{GrMod} A / \text { Fdim } A,
$$

and denote by $\pi$ and $\omega$ the quotient functor and its right adjoint. The projective space $X$ with homogeneous coordinate ring $A$ is defined by $\operatorname{Mod} X:=$ Tails $A$.

The structure module of $X$ is $\mathcal{O}_{X}:=\pi A$. We define

$$
\operatorname{Proj} A=\left(X, \mathcal{O}_{X}\right) \text {. }
$$

$\operatorname{Proj} A$ is an enriched space in the sense of [25. Section 3.6].

The cohomology groups of a module $\mathcal{F}$ over the enriched space $X$ are defined in [27] and [3] to be

$$
H^{q}(X, \mathcal{F}):=\operatorname{Ext}_{X}^{q}\left(\mathcal{O}_{X}, \mathcal{F}\right)
$$

The idea for the next definition is due to Rosenberg [21, Section 1]. 
Definition 2.3. If $X$ and $Z$ are non-commutative spaces, a weak map $f: Z \rightarrow X$ is a natural equivalence class of left exact functors $f_{*}: \operatorname{Mod} Z \rightarrow \operatorname{Mod} X$. A weak map $f: Z \rightarrow X$ is a map if $f_{*}$ has a left adjoint. A weak map is affine [21] Section 4.3] if $f_{*}$ is faithful, and has both a left and a right adjoint. A left adjoint to $f_{*}$ will be denoted by $f^{*}$, and a right adjoint will be denoted by $f^{!}$.

A weak map $f$ is a map if and only if $f_{*}$ commutes with products.

Let $f: X \rightarrow Y$ be a morphism of schemes. Suppose that Qcoh $X$ and Qcoh $Y$ are Grothendieck categories, so that $X$ and $Y$ are spaces in our sense. If either $X$ is noetherian, or $f$ is quasi-compact and separated, then $f$ induces a map in the sense of Definition 2.3 10, Proposition 5.8, Ch. II]. However, there are maps that are not induced by morphisms. For example, the inclusion

$$
\left(\begin{array}{ll}
k & 0 \\
0 & k
\end{array}\right) \rightarrow\left(\begin{array}{ll}
k & k \\
k & k
\end{array}\right)
$$

induces an affine map $\operatorname{Spec} k \rightarrow \operatorname{Spec}(k \times k)$ of spaces that is not induced by a morphism between these schemes.

Definition 2.4. Let $X$ be a non-commutative space. A weakly closed subspace of $X$, say $W$, is a full subcategory $\operatorname{Mod} W$ of $\operatorname{Mod} X$ that is closed under subquotients (and therefore under isomorphisms), and for which the inclusion functor $\alpha_{*}: \operatorname{Mod} W \rightarrow \operatorname{Mod} X$ has a right adjoint, denoted by $\alpha$. We write $\alpha: W \rightarrow X$ for the weak map corresponding to $\alpha_{*}$. A weakly closed subspace $W$ is closed if $\alpha_{*}$ also has a left adjoint; we will denote a left adjoint to $\alpha_{*}$ by $\alpha^{*}$.

For example, $X$ and $\phi$ are closed subspaces of $X$.

The inclusion of a closed subspace is an affine map.

Let $W$ be a weakly closed subspace of $X$ and let $\alpha: W \rightarrow X$ be the inclusion. Then $\operatorname{Mod} W$ is a Grothendieck category, and is locally noetherian if $\operatorname{Mod} X$ is. Because $\operatorname{Mod} W$ is closed under subquotients, $\alpha_{*}$ is an exact functor. Because $\alpha^{!}$ has an exact left adjoint, it sends injective $X$-modules to injective $W$-modules. The definitions imply that $\alpha^{!} \alpha_{*} \cong \operatorname{id}_{W}$ when $W$ is a weakly closed, and $\alpha^{*} \alpha_{*} \cong \operatorname{id}_{W}$ when $W$ is closed.

Let $i: Z \rightarrow X$ be the inclusion of a closed subscheme of a scheme $X$. One often blurs the distinction between the structure sheaf $\mathcal{O}_{Z}$, which lies in Qcoh $Z$, and its direct image $i_{*} \mathcal{O}_{Z}$, which lies in Qcoh $X$. We will do something similar when $W$ is a weakly closed subspace of a space $X$. If $\alpha: W \rightarrow X$ is the inclusion, we will confuse the trivial $W$-W-bimodule $o_{W}$ with the weak $X$-X-bimodule corresponding to the left exact functor $\alpha_{*} \alpha^{!}$. Thus, we will speak of the map $o_{X} \rightarrow o_{W}$ of weak $X-X$ bimodules, the kernel of which is the weak ideal defining $W$. For more information about weak ideals and bimodules, see [25].

Definition 2.5. Let $X$ be a non-commutative space. A weakly open subspace of $X$, say $U$, is a full subcategory $\operatorname{Mod} U$ of $\operatorname{Mod} X$ that is closed under isomorphism and is such that the inclusion functor $j_{*}: \operatorname{Mod} U \rightarrow \operatorname{Mod} X$ has an exact left adjoint $j^{*}$. If $V$ is another weakly open subspace of $X$, we say that $U$ is contained in $V$, and write $U \subset V$, if $\operatorname{Mod} U \subset \operatorname{Mod} V$.

If a weakly closed subspace is also a weakly open subspace, then it is a closed subspace. 
Definition 2.6. We call a weak map $f: Y \rightarrow X$ a closed immersion (resp., weakly closed immersion, weakly open immersion, open immersion) if it is an isomorphism onto a closed (resp. weakly closed, weakly open, open) subspace of $X$.

The next two results are standard: they follow from the fact that $\alpha^{!}$and $j_{*}$ are right adjoints to exact functors and so preserve injectives.

Lemma 2.7. Let $\alpha: W \rightarrow X$ be a weakly closed immersion. If $M \in \operatorname{Mod} W$ and $N \in \operatorname{Mod} X$, then there is a spectral sequence

$$
\operatorname{Ext}_{W}^{p}\left(M, R^{q} \alpha^{!} N\right) \Rightarrow \operatorname{Ext}_{X}^{p+q}\left(\alpha_{*} M, N\right) .
$$

Lemma 2.8. If $j: U \rightarrow X$ is a weakly open immersion, then there is a spectral sequence

$$
\operatorname{Ext}_{X}^{p}\left(M, R^{q} j_{*} N\right) \Rightarrow \operatorname{Ext}_{U}^{p+q}\left(j^{*} M, N\right)
$$

whenever $M \in \operatorname{Mod} X$ and $N \in \operatorname{ModU}$.

\section{Weakly ClOSED SUBSPaCES}

Rosenberg has shown that the closed subspaces of an affine space are in bijection with the two-sided ideals of its coordinate ring. A typical non-commutative ring has few two-sided ideals, and a typical non-commutative space has few closed subspaces. A more useful notion for non-commutative geometry is that of a weakly closed subspace.

This section shows that there are reasonable notions of intersection and union for weakly closed subspaces.

The notion of a weakly closed subspace makes sense for schemes. For example, Spec $\mathbb{Z}$ has a weakly closed subspace $W$ defined by declaring Mod $W$ to consist of the torsion abelian groups. There is a proper descending chain of weakly closed subspaces $W=W_{0} \supset W_{1} \supset W_{2} \supset \ldots$ defined by declaring Mod $W_{i}$ to be all torsion groups that have no torsion with respect to the first $i$ positive primes.

If $X$ is a scheme such that Qcoh $X$ is a Grothendieck category, and $Z$ is a closed subscheme of $X$, then the full subcategory of Qcoh $X$ consisting of the modules whose support lies in $Z$ is closed under subquotients and direct limits, so it is the module category of a weakly closed subspace of $X$; this subspace is rarely closed.

Let $X$ be a space and $M$ an $X$-module. Let $W$ be the smallest weakly closed subspace such that $M \in \operatorname{Mod} W$. Then $\operatorname{Mod} W$ consists of all subquotients of all direct sums of copies of $M$. As a subcategory of $\operatorname{Mod} X, \operatorname{Mod} W$ is closed under subquotients, so the inclusion functor is exact; it is closed under direct sums because direct sums are exact in $\operatorname{Mod} X$; it is therefore closed under direct limits, so the inclusion functor has a right adjoint.

The following is a triviality.

Lemma 3.1. If $Z$ is a weakly closed subspace of $X$ and $W$ is a weakly closed subspace of $Z$, then $W$ is a weakly closed subspace of $X$.

Definition 3.2. Let $Z$ and $W$ be weakly closed subspaces of $X$. We say that $Z$ lies on $W$, or is contained in $W$, if $\operatorname{Mod} Z$ is contained in $\operatorname{Mod} W$; we write $Z \subset W$ to indicate this. Their intersection $W \cap Z$ is defined by declaring that

$$
\operatorname{Mod} W \cap Z:=\operatorname{Mod} W \cap \operatorname{Mod} Z \text {. }
$$

That is, $\operatorname{Mod} W \cap Z$ is the full subcategory of $\operatorname{Mod} X$ consisting of those $X$ modules that belong to both $\operatorname{Mod} W$ and $\operatorname{Mod} Z$. 
Proposition 3.3. Suppose that $W$ and $Z$ are weakly closed subspaces of $X$. Then

1. $W$ and $Z$ are spaces;

2. $W \cap Z$ is weakly closed in $X$, and in $W$, and in $Z$;

3. if $Z$ is closed in $X$, then $W \cap Z$ is closed in $W$;

4. if $W$ and $Z$ are closed in $X$, then $W \cap Z$ is closed in $X$, and in $W$, and in $Z$;

5. if $Z \subset W$, then $Z$ is a weakly closed subspace of $W$;

6. if $Z \subset W$ and $Z$ is closed in $X$, then $Z$ is closed in $W$;

7. if $Z$ is also weakly open in $X$, then $W \cap Z$ is both closed and weakly open in $W$.

Proof. Let $\alpha: W \rightarrow X$ and $\beta: Z \rightarrow X$ be the inclusions. Let $\gamma_{*}: \operatorname{Mod} W \cap Z \rightarrow$ $\operatorname{Mod} Z$ and $\delta_{*}: \operatorname{Mod} W \cap Z \rightarrow \operatorname{Mod} W$ be the inclusion functors.

(1) To see that $W$ is a space one checks that $\operatorname{Mod} W$ is a Grothendieck category. By, for example, [25. Proposition 3.4.3], ModW is a cocomplete abelian category, and the inclusion $\alpha_{*}$ is exact and commutes with direct limits. Since direct limits in $\operatorname{Mod} W$ coincide with direct limits in $\operatorname{Mod} X, \operatorname{Mod} W$ has exact direct limits.

Since $\operatorname{Mod} X$ has a small set of generators, the collection of all submodules of any $X$-module is a small set, and hence so is the collection of all its subquotients. If $\left\{M_{i} \mid i \in I\right\}$ is a small set of generators for $\operatorname{Mod} X$, then the set of all subquotients of all $M_{i}$ that belong to ModW is small. This is a set of generators for ModW: if $f: L \rightarrow N$ is a non-zero morphism in ModW, then there is a morphism $g: M_{i} \rightarrow L$ such that $f g \neq 0$; since $L \in \operatorname{Mod} W, g$ induces a morphism $g^{\prime}: M^{\prime} \rightarrow L$ from some subquotient $M^{\prime}$ of $M_{i}$ belonging to $\operatorname{Mod} W$ such that $f g^{\prime} \neq 0$.

(2) Since $\operatorname{Mod} W$ and $\operatorname{Mod} Z$ are both closed under subquotients and direct limits in $\operatorname{Mod} X$, so is $\operatorname{Mod} W \cap Z$. It follows that $\operatorname{Mod} W \cap Z$ is an abelian category and that its inclusion in $\operatorname{Mod} X$ is an exact functor commuting with direct limits. The inclusion functor therefore has a right adjoint. This proves (2).

Hence there is a commutative diagram

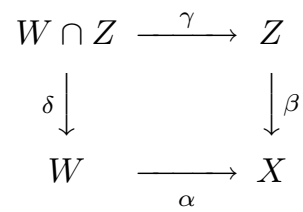

of spaces and weak maps. Since $\alpha_{*}, \beta_{*}, \gamma_{*}$ and $\delta_{*}$, are inclusions of subcategories, $\alpha_{*} \delta_{*}=\beta_{*} \gamma_{*}$. Up to natural equivalence we also have $\delta^{!} \alpha^{!}=\gamma^{!} \beta^{!}$.

(3) To see that $W \cap Z$ is closed in $W$ it suffices to show that $\delta_{*}$ commutes with products; that is, that $\operatorname{Mod} W \cap Z$ is closed under products in ModW. Let $M_{l}$ be a family in $\operatorname{Mod} Z \cap W$, and suppose they have a product in $\operatorname{Mod} W$. That product is $\alpha^{!}\left(\prod M_{l}\right)$, where $\prod M_{l}$ is their product in $\operatorname{Mod} X$. But $\operatorname{Mod} Z$ is closed under products in $\operatorname{Mod} X$, so contains $\prod M_{l}$, and hence its submodule $\alpha^{!}\left(\prod M_{l}\right)$. Therefore $\alpha^{!}\left(\prod M_{l}\right)$ is in $\operatorname{Mod} W \cap Z$. This proves that $\delta_{*}$ has a left adjoint $\delta^{*}$, whence $W \cap Z$ is closed in $W$.

We will now show that

$$
\gamma_{*} \delta^{*} \cong \beta^{*} \alpha_{*}
$$

Since $\gamma_{*} \delta^{*}$ is a left adjoint to $\delta_{*} \gamma^{!}$and $\beta^{*} \alpha_{*}$ is a left adjoint to $\alpha^{!} \beta_{*}$, it suffices to show that $\delta_{*} \gamma^{!} \cong \alpha^{!} \beta_{*}$. If $M$ is a $Z$-module, $\delta_{*} \gamma^{!} M$ is the largest submodule of $M$ that is in $\operatorname{Mod} Z \cap \operatorname{Mod} W$; since every submodule of $M$ is in $\operatorname{Mod} Z, \delta_{*} \gamma^{!} M$ is the 
largest submodule of $M$ that is in $\operatorname{Mod} W$; but that submodule is $\alpha^{!} \beta_{*} M$. It follows that

$$
\delta_{*} \gamma^{!} \cong \alpha^{!} \beta_{*},
$$

and (3.2) now follows.

(4) If $W$ and $Z$ are closed subspaces of $X$, then $\operatorname{Mod} W$ and $\operatorname{Mod} Z$ are closed under products in $\operatorname{Mod} X$. It follows that $\operatorname{Mod} W \cap Z$ is closed under products in $X$, whence $W \cap Z$ is closed.

(5) and (6) follow from (2) and (3) because $Z \subset W$ implies that $W \cap Z=Z$.

(7) Since $Z$ is both weakly closed and weakly open in $X, \beta_{*}$ has an exact left adjoint $\beta^{*}$. In particular, $Z$ is closed in $X$. Hence by (3), $W \cap Z$ is closed in $W$, and $\gamma_{*} \delta^{*} \cong \beta^{*} \alpha_{*}$. Since $\beta^{*} \alpha_{*}$ is a composition of exact functors, $\gamma_{*} \delta^{*}$ is exact. It follows that $\delta^{*}$ is exact, because $\gamma$ is a weakly closed immersion.

Remarks. 1. The following two observations are easily checked. If $W$ is a weakly closed subspace of $Z$, and $Z$ is a weakly closed subspace of $X$, then $W$ is a weakly closed subspace of $X$. If $W$ is a closed subspace of $Z$, and $Z$ is a closed subspace of $X$, then $W$ is a closed subspace of $X$.

2. The binary operation $\cap$ on weakly closed subspaces is idempotent, commutative, and associative. One has $W \cap \phi=\phi$ and $W \cap X=W$.

3. One may define the intersection of an arbitrary collection of weakly closed subspaces $W_{i}$ by $\operatorname{Mod}\left(\bigcap W_{i}\right):=\bigcap \operatorname{Mod} W_{i}$. It is clear that $\bigcap W_{i}$ is closed in $\operatorname{Mod} X$ under subquotients and direct limits, because each $\operatorname{Mod} W_{i}$ is. Hence $\bigcap_{i} W_{i}$ is a weakly closed subspace of $X$ (cf. [20, Lemma 6.2.2, Chapter 3]).

Definition 3.4. Let $W_{i}, i \in I$, be a finite collection of weakly closed subspaces of $X$. Their union, denoted $\bigcup_{i} W_{i}$, is the smallest weakly closed closed subspace of $X$ that contains all the $W_{i}$. It is the intersection of all the weakly closed subspaces that contain all the $W_{i}$.

Remarks. 1. We could first have defined the union of a pair of weakly closed subspaces, thus making $\cup$ a binary operation. One sees that $U$ is idempotent, commutative, and associative. Associativity could then be used to define arbitrary finite unions; this definition would agree with the one in Definition 3.4. It is clear that $W \cup \phi=W$ and $W \cup X=X$.

2. If $W$ and $Z$ are weakly closed, then $\operatorname{Mod} W \cup Z$ is the full subcategory consisting of all subquotients of modules of the form $M \oplus N$, where $M \in \operatorname{Mod} W$ and $N \in \operatorname{Mod} Z$. Obviously $\operatorname{Mod} W \cup Z$ is closed under subquotients; it is closed under direct sums because $\operatorname{Mod} W$ and $\operatorname{Mod} Z$ are, and because direct sums are exact in $\operatorname{Mod} X$; it is therefore closed under direct limits, so is weakly closed; it contains $\operatorname{Mod} W$ and $\operatorname{Mod} Z$, and any weakly closed subcategory containing $\operatorname{Mod} W$ and $\operatorname{Mod} Z$ must contain all modules of the form $M \oplus N$; hence this category equals $\operatorname{Mod} W \cup Z$.

3. If $X$ is the affine space with coordinate $\operatorname{ring} R$, and $W$ and $Z$ are the closed subspaces cut out by the two-sided ideals $I$ and $J$, then $W \cup Z$ is the closed subspace cut out by $I \cap J$. To see this, first observe that $\operatorname{Mod} R / I \cap J$ is weakly closed and contains both $\operatorname{Mod} R / I$ and $\operatorname{Mod} R / J$; secondly, any weakly closed subspace that contains both $W$ and $Z$ must contain $R / I \oplus R / J$, and hence its submodule $R / I \cap J$, and therefore must contain $\operatorname{Mod} R / I \cap J$.

4. Let $W$ and $Z$ be weakly closed subspaces of $X$. Their Gabriel product $W \bullet Z$ is the weakly closed subspace defined by declaring $\operatorname{Mod} W \bullet Z$ to be the full subcategory 
of $\operatorname{Mod} X$ consisting of the modules $M$ for which there is an exact sequence $0 \rightarrow$ $L \rightarrow M \rightarrow N \rightarrow 0$ with $N \in \operatorname{Mod} W$ and $L \in \operatorname{Mod} Z$ [6], p. 395]. Van den Bergh has shown that $W \bullet Z$ is weakly closed, and is closed if both $Z$ and $W$ are [25, Prop. 3.4.5]. Since $W \bullet Z$ contains both $W$ and $Z$, it follows that

$$
W \cup Z \subset W \bullet Z \cap Z \bullet W .
$$

In the situation of the previous remark, $\operatorname{Mod}(W \bullet Z \cap Z \bullet W)=\operatorname{Mod} R / I J+J I$, so in general the inclusion in (3.4) is strict. Example 6.11 exhibits closed points $p$ and $q$ in a an affine space such that $p \cup q=p \bullet q \neq q \bullet p$.

5. If $W, Y$ and $Z$ are weakly closed subspaces of $X$, we do not know whether $W \cap(Y \cup Z)=(W \cap Y) \cup(W \cap Z)$. The analogous equality for weakly open subspaces does hold (Lemma 6.16).

\section{Closed subspaces}

The first evidence that the notion of a closed subspace in Definition 2.4 is appropriate for non-commutative geometry is the following result of Rosenberg [20] Proposition 6.4.1, p.127]. If $X$ is an affine space, say $X=\operatorname{Mod} R$, then each twosided ideal $I$ cuts out a closed subspace of $X$, namely $\operatorname{Mod} R / I$, and this sets up a bijection between the closed subspaces of $X$ and the two-sided ideals of $R$ (see also [22, Proposition 2.3]). We call the closed subspace $\operatorname{Mod} R / I$ the zero locus of $I$. Thus, if $X$ is an affine scheme, its closed subschemes are in natural bijection with its closed subspaces. We prefer to say that the closed subspaces are the same things as the closed subschemes. Further evidence is provided by 24, Theorem $3.2]$, which shows that a two-sided ideal in an $\mathbb{N}$-graded $k$-algebra $A$ cuts out a closed subspace of $\operatorname{Proj} A$. There is further evidence. If $X$ is a scheme such that Qcoh $X$ is a Grothendieck category (for example, if $X$ is noetherian) and $Z$ is a closed subscheme of $X$, then $\mathrm{Q} \operatorname{coh} Z$ is a closed subspace of $\mathrm{Qcoh} X$ in the sense of Definition 2.4. The main result in this section (Theorem 4.1) shows that if $X$ is a quasi-projective scheme over a field $k$, then the closed subschemes of $X$ are the same as the closed subspaces of $X$.

In this section we also prove a non-commutative geometric version of Matlis' result on the bijection between prime ideals in a commutative noetherian ring and indecomposable injectives over that ring (Proposition 4.7).

Remarks. 1. An interesting and ubiquitous non-commutative space is the graded line $\mathbb{L}^{1}$, defined by $\operatorname{Mod} \mathbb{L}^{1}=\operatorname{GrMod} k[x]$ where $k$ is a field and $\operatorname{deg} x=1$. We associate to every subset $D \subset \mathbb{Z}$ a closed subspace $Z_{D}$ of $\mathbb{L}^{1}$ by declaring $\operatorname{Mod} Z_{D}$ to be the full subcategory consisting of those graded $k[x]$-modules $M$ such that $M_{n}=0$ for all $n \notin D$. Since there is a proper descending chain of subsets $D, \mathbb{L}^{1}$ does not have the descending chain condition on closed subspaces. If $D$ consists of $n$ consecutive integers, then $Z_{D}$ is isomorphic to the affine space with coordinate ring the $n \times n$ upper triangular matrices over $k$.

If $X$ is an affine space with coordinate ring the enveloping algebra of a nonabelian solvable Lie algebra over a field of characteristic zero, then $X$ contains many weakly closed subspaces isomorphic to $\mathbb{L}^{1}[22$. If $\tilde{X}$ is the blowup of a noncommutative surface $X$ at a closed point $p$ lying on an effective divisor $Y$ such that $\left\{\mathcal{O}_{p}(n Y) \mid n \in \mathbb{Z}\right\}$ is infinite, then the exceptional curve on $\tilde{X}$ is a weakly closed subspace of $\tilde{X}$ isomorphic to $\mathbb{L}^{1}[25]$. 
2. The category of right comodules over a coalgebra is a Grothendieck category, so it is a space in the sense of Definition 2.1. Let $C$ and $D$ be coalgebras and let $X$ and $Y$ be the spaces defined by $\operatorname{Mod} X=\operatorname{Comod} C$ and $\operatorname{Mod} Y=\operatorname{Comod} D$. If $\varphi$ : $C \rightarrow D$ is a homomorphism of coalgebras, then the rule $(M, \rho) \mapsto\left(M,\left(\operatorname{id}_{M} \otimes \varphi\right) \rho\right)$ gives an exact functor $f_{*}: \operatorname{Comod} C \rightarrow \operatorname{Comod} D$ that is the direct image functor for an affine map $f: X \rightarrow Y$. The right adjoint to $f_{*}$ is $f^{!}=-\square_{D} C$, the cotensor product. Joyal and Street [14, Proposition 2, p. 454] show that the closed subspaces of $Y$ are in bijection with the subcoalgebras of $D$. Actually they don't quite prove that, but using the fact that in a locally finite space weakly closed and closed subspaces coincide (Proposition 4.8), their result implies this. The closed points of $Y$, in the sense of Section 5 are in bijection with the simple subcoalgebras of $D$.

Theorem 4.1. Let $X$ be a noetherian scheme having an ample line bundle (for example, let $X$ be quasi-projective over Spec $k$ with $k$ a field). Then closed subschemes and closed subspaces of $X$ are the same things.

We will prove the theorem after the next lemma.

Let $\mathcal{O}_{X}(1)$ denote an ample line bundle on $X$. If $i: Z \rightarrow X$ is the inclusion of a closed subscheme, we write $\mathcal{O}_{Z}(1)$ for its restriction, $i^{*}\left(\mathcal{O}_{X}(1)\right)$, to $Z$. Thus $\mathcal{O}_{Z}(1)$ is an ample line bundle on $Z$.

Let $\mathcal{M}$ be an $\mathcal{O}_{X}$-module. Its annihilator, which we denote by $\operatorname{Ann} \mathcal{M}$, is the kernel of the natural map $\mathcal{O}_{X} \rightarrow \mathcal{H o m}_{\mathcal{O}_{X}}(\mathcal{M}, \mathcal{M})$. Clearly Ann $\mathcal{M}$ is the largest ideal $\mathcal{I}$ in $\mathcal{O}_{X}$ such that $\mathcal{M I}=0$, where $\mathcal{M I}$ is defined as the image of the natural map $\mathcal{M} \otimes_{\mathcal{O}_{X}} \mathcal{I} \rightarrow \mathcal{M}$. If $\mathcal{M}$ is coherent, its scheme-theoretic support is the closed subscheme of $X$ cut out by its annihilator, i.e., it is $\left(Z, \mathcal{O}_{Z}\right)$, where $\mathcal{O}_{Z}=\mathcal{O}_{X} / \operatorname{Ann} \mathcal{M}$.

Lemma 4.2. Let $\mathcal{M}$ be a coherent $\mathcal{O}_{X}$-module, and $Z$ its scheme-theoretic support. If $W$ is a weakly closed subspace of $X$ such that $\mathcal{M} \in \operatorname{Mod} W$, then $Z \subset W$, i.e., QcohZ $\subset \operatorname{Mod} W$.

Proof. We show first that some finite direct sum of copies of $\mathcal{M}$ has a submodule that is an invertible $\mathcal{O}_{Z}$-module.

Since $\mathcal{M}$ is a coherent $\mathcal{O}_{Z}$-module, there is an epimorphism $\bigoplus \mathcal{O}_{Z}(-n) \rightarrow \mathcal{M}$ for some $n \gg 0$ and some suitable finite direct sum. The image of each $\mathcal{O}_{Z}(-n)$ in $\mathcal{M}$ is isomorphic to $\left(\mathcal{O}_{Z} / \mathcal{I}_{j}\right)(-n)$ for some coherent ideal $\mathcal{I}_{j}$ in $\mathcal{O}_{Z}$. Since $\mathcal{M}$ is the sum of these images, the annihilator of $\mathcal{M}$ in $\mathcal{O}_{Z}$ is the intersection of all the $\mathcal{I}_{j}$. By definition of $Z$, that annihilator is zero, so the diagonal map $\mathcal{O}_{Z} \rightarrow \bigoplus \mathcal{O}_{Z} / \mathcal{I}_{j}$ is monic. It follows that $\mathcal{O}_{Z}(-n)$ embeds in a finite direct sum of copies of $\mathcal{M}$.

By the preceding paragraph, $\mathcal{O}_{Z}(-n)$ belongs to $\operatorname{Mod} W$ for some $n$, and hence for all $n \gg 0$. Since every coherent $\mathcal{O}_{Z}$-module is a quotient of a suitable finite direct sum of copies of $\mathcal{O}_{Z}(-n)$ for some sufficiently large $n, \operatorname{coh} Z \subset \operatorname{Mod} W$. Since $\operatorname{Mod} W$ is closed under direct limits, it follows that $\mathrm{Q} \operatorname{coh} Z \subset \operatorname{Mod} W$.

Proof of Theorem 4.1. Let $W$ be a closed subspace of $X$. We must show that $\operatorname{Mod} W=\mathrm{Q} \operatorname{coh} Z$ for some closed subscheme $Z$ of $X$. Let $\alpha: W \rightarrow X$ be the inclusion. The counit $\mathcal{O}_{X} \rightarrow \alpha_{*} \alpha^{*} \mathcal{O}_{X}$ is an epimorphism, so $\alpha_{*} \alpha^{*} \mathcal{O}_{X}=\mathcal{O}_{Z}$ for a unique closed subscheme $Z$ of $X$. Since $\mathcal{O}_{Z}$ is a $W$-module, Lemma 4.2 shows that Qcoh $Z \subset \operatorname{Mod} W$.

Suppose that $\mathcal{F} \in \bmod W$, and let $Y$ denote its scheme-theoretic support. By Lemma 4.2, Qcoh $Y \subset \operatorname{Mod} W$. Since $Y$ is also the scheme-theoretic support of 
$\mathcal{F}(n), \mathcal{F}(n) \in \operatorname{Mod} W$ for all $n \in \mathbb{Z}$. For some $n \gg 0$ and some suitably large finite direct sum, there is an epimorphism $\bigoplus \mathcal{O}_{X} \rightarrow \mathcal{F}(n)$, hence an epimorphism

$$
\bigoplus \alpha_{*} \alpha^{*} \mathcal{O}_{X} \rightarrow \alpha_{*} \alpha^{*}(\mathcal{F}(n)) \cong \mathcal{F}(n) .
$$

It follows that $\mathcal{F}(n)$ is in $\mathrm{Qcoh} Z$. Hence $\mathcal{F} \in \mathrm{Q} \operatorname{coh} Z$ also, and we conclude that $\operatorname{Mod} W=\mathrm{Q} \operatorname{coh} Z$, as desired.

This result suggests the following definitions.

Definition 4.3. Let $X$ be a locally noetherian space. The support of a noetherian $X$-module $M$ is the smallest closed subspace $Z$ such that $M \in \operatorname{Mod} Z$. (Such a smallest closed subspace exists because an intersection of closed subspaces is closed.) A non-zero $X$-module is prime if all its non-zero submodules have the same support. A non-empty closed subspace $Z$ of $X$ is prime if it is the support of a prime noetherian $X$-module.

Our definition of a prime module is modelled on the analogous notion for modules over rings - see, for example, [17, Section 4.3.4]. Our definition of prime module in terms of support emerged in discussions with C. Pappacena and agrees with his definition in [18].

If $X$ has the descending chain condition on closed subspaces, then every nonzero $X$-module has a prime submodule: simply take a submodule whose support is as small as possible. As remarked at the beginning of this section, the graded line, GrModk $k x]$, does not have the descending chain condition on closed subspaces; it is easy to see that $k[x]$, as an object in $\operatorname{GrMod} k[x]$, does not contain a prime submodule.

Let $X$ be an affine space with coordinate ring $R$. The support of an $R$-module is the zero locus of its annihilator because $R / \operatorname{Ann} M$ embeds in a product of copies of $M$, and so it is a module over any closed subspace that contains $M$. A closed subspace of $X$ is prime if and only if it is the zero locus of a prime ideal; thus prime subspaces of $X$ are in bijection with the prime ideals in $R$.

A non-zero submodule of a prime module is prime. A direct sum of copies of a prime module is prime. An auto-equivalence of $\operatorname{Mod} X$ sends prime modules to prime modules.

By Lemma 4.2 if $X$ is a noetherian scheme having an ample line bundle, the support as we have just defined it is the same as the scheme-theoretic support.

Proposition 4.4. Let $X$ be a noetherian scheme having an ample line bundle.

1. The (scheme-theoretic) support of a prime module is integral.

2. A closed subscheme $Z \subset X$ is integral if and only if $\mathcal{O}_{Z}$ is prime.

Proof. We will use the fact that a closed subscheme $Z$ of $X$ is reduced and irreducible if and only if the product of two non-zero ideals of $\mathcal{O}_{Z}$ is non-zero.

(1) Let $Z$ denote the scheme-theoretic support of a prime module $\mathcal{M}$. Without loss of generality we may assume that $\mathcal{M}$ is coherent. By the arguments in Lemma 4.2. $\mathcal{O}_{Z}(-n)$ embeds in a finite direct sum of copies of $\mathcal{M}$. It follows that $\mathcal{O}_{Z}(-n)$, and hence $\mathcal{O}_{Z}$, is prime. Hence, the scheme-theoretic support of every non-zero coherent ideal of $\mathcal{O}_{Z}$ is $Z$. In other words, if $\mathcal{I}$ is a non-zero coherent ideal of $\mathcal{O}_{Z}$, then $\mathcal{I} \mathcal{J} \neq 0$ for all non-zero ideals $\mathcal{J}$ of $\mathcal{O}_{Z}$. Hence $Z$ is reduced and irreducible.

(2) If $Z$ is integral, then every non-zero ideal of $\mathcal{O}_{Z}$ has annihilator equal to zero, so it has scheme-theoretic support $Z$, thus showing that $\mathcal{O}_{Z}$ is prime. Conversely, 
if $\mathcal{O}_{Z}$ is prime, then every non-zero ideal of $\mathcal{O}_{Z}$ has support equal to $Z$ and so has zero annihilator, thus showing that $Z$ is integral.

The conclusion of Lemma 4.2 captures one of the essential aspects of commutativity: it says that $X$ has a lot of closed subspaces. This is similar to saying that a ring has lots of two-sided ideals. The next definition singles out this property, and we think of it as saying that the space is "almost commutative".

Definition 4.5. A locally noetherian space $X$ has enough closed subspaces if every noetherian module $M$ has the following property: the smallest weakly closed subspace $W \subset X$ such that $M \in \operatorname{Mod} W$ is equal to the support of $M$.

In other words, $X$ has enough closed subspaces if every weakly closed subspace $W \subset X$ has the following property: if $M$ is a noetherian $W$-module, then there is a closed subspace $Z$ of $X$ such that $Z \subset W$ and $M \in \operatorname{Mod} Z$. Lemma 4.2 implies that a quasi-projective scheme has enough closed subspaces.

The next result shows that this notion of "almost commutative" is compatible with an older idea of what it means for a ring to be "almost commutative". Gabriel's condition (H) 6, page 422] is expressed as part (2) of the next result; Gabriel (loc. cit., page 423) showed that (2) implies (3) and Cauchon [5] proved the converse. The equivalent condition (1) is geometric and therefore has meaning for non-affine spaces. For example, if $A$ is a connected graded noetherian algebra that is a finite module over its center, then property (1) is satisfied by $\operatorname{Proj} A$.

Proposition 4.6. Let $X$ be a locally noetherian affine space with coordinate ring $R$. The following are equivalent:

1. $X$ has enough closed subspaces;

2. if $M$ is a noetherian $R$-module, then $\operatorname{Ann} M=\bigcap_{i=1}^{n} \operatorname{Ann}\left(m_{i}\right)$ for some finite set of elements $m_{i} \in M$;

3. there is a bijection

$\{$ prime ideals in $R\} \leftrightarrow\{$ iso-classes of indecomposable injective $R$-modules $\}$

given by $\mathfrak{p} \mapsto$ the injective envelope of $R / \mathfrak{p}$, and $E \mapsto$ the largest ideal annihilating a non-zero submodule of $E$.

Proof. Gabriel [6, page 423] and Cauchon [5] have proved the equivalence of (2) and (3).

$(2) \Rightarrow(1)$ Let $M$ be a noetherian $X$-module, and let $W$ be a weakly closed subspace containing $M$. Set $I=\operatorname{Ann} M$, and let $Z$ be the zero locus of $I$. There are elements $m_{i} \in M, 1 \leq i \leq n$, such that the diagonal map $R / I \rightarrow \bigoplus_{i=1}^{n} m_{i} R$ is injective. Since $R / I$ embeds in a finite direct sum of copies of $M, R / I \in \operatorname{Mod} W$, whence $Z \subset W$. Since $M$ is a $Z$-module, this shows that $X$ has enough closed subspaces.

$(1) \Rightarrow(2)$ Let $M$ be a noetherian $R$-module with support $Z$. The smallest weakly closed subspace of $X$ containing $M$ is $W$, where $\operatorname{Mod} W$ consists of all subquotients of direct sums of copies of $M$. By hypothesis, $R / \operatorname{Ann} M$ is in $\operatorname{Mod} W$, so it is a subquotient of a direct sum of copies of $M$. Since $R / \operatorname{Ann} M$ is a finitely generated projective module over itself, it follows that $R / \operatorname{Ann} M$ is a submodule of a finite direct sum of copies of $M$. If $\varphi: R / \operatorname{Ann} M \rightarrow M^{\oplus n}$ is injective, and $m_{i}$ denotes the image of $\varphi(1)$ under the $i^{\text {th }}$ projection, then Ann $M=\bigcap_{i=1}^{n} \operatorname{Ann}\left(m_{i}\right)$. Thus (2) holds. 
Rings satisfying condition (2) are said to be fully bounded - see [7, Chapter 8] and [17, Sections 6.4 and 6.10.4] for more information about this notion. The standard example of such a ring is one that is a finite module over its center.

In the next proof we use the notion of Krull dimension as defined by Gabriel. A noetherian $X$-module is critical if $\operatorname{Kdim} M / N<\operatorname{Kdim} M$ for all non-zero submodules $N \subset M$. The definition of Krull dimension is such that every non-zero module has a non-zero critical submodule. A submodule of a critical module is critical. Hence a prime subspace is the support of a critical module, and that module may also be chosen to be prime and noetherian.

Proposition 4.7. Let $X$ be a locally noetherian space having the descending chain condition on closed subspaces. If $X$ has enough closed subspaces, there is a bijection $\{$ prime subspaces of $X\} \longleftrightarrow\{$ iso-classes of indecomposable injective $X$-modules $\}$ given by $Z \mapsto$ the injective envelope of a critical noetherian prime module having support equal to $Z$, and $E \mapsto$ the support of a critical prime noetherian submodule of $E$.

Proof. Let $Z$ be a prime subspace of $X$ and let $M$ be a critical noetherian prime module having support equal to $Z$. Since $M$ is critical it is uniform, so its injective envelope, say $E$, is indecomposable. We will show that the rule $Z \mapsto E$ is a welldefined map setting up the claimed bijection.

To show the map is well-defined, we must show that $E \cong E^{\prime}$ whenever $E^{\prime}$ is an injective envelope of another critical noetherian prime module, say $M^{\prime}$, whose support is equal to $Z$.

The hypothesis on $X$ implies that $\operatorname{Mod} Z$ consists of all subquotients of all direct sums of copies of $M$. Of course, the same is true with $M^{\prime}$ in place of $M$. Since each of $M$ and $M^{\prime}$ is a subquotient of a finite direct sum of copies of the other, they have the same Krull dimension.

Choose the smallest integer $n$ such that $M^{\prime}$ is isomorphic to $L / K$, where $K \subset$ $L \subset M_{1} \oplus \ldots \oplus M_{n}$ and each $M_{i} \cong M$. The minimality of $n$ ensures that $L \cap M_{i}$ is non-zero for all $i$. If $K \cap M_{i}$ were non-zero for all $i$, then the Krull dimension of $L / K$ would be strictly smaller than that of $M$; this is not the case, so $K \cap M_{i}$ is zero for some $i$. Hence $L \cap M_{i}$ embeds in $M^{\prime}$. In particular, $M$ and $M^{\prime}$ have a common non-zero submodule, from which we conclude that $E \cong E^{\prime}$. Hence the map $Z \mapsto E$ is well-defined.

To see that the map $Z \mapsto E$ is injective, suppose that $Z$ and $Z^{\prime}$ are prime subspaces for which the corresponding indecomposable injectives, say $E$ and $E^{\prime}$, are isomorphic. Let $M$ and $M^{\prime}$ be critical noetherian prime modules whose supports are $Z$ and $Z^{\prime}$ respectively. Thus $E$ and $E^{\prime}$ are injective envelopes of $M$ and $M^{\prime}$ respectively. Since $E$ and $E^{\prime}$ are isomorphic and indecomposable, $M$ and $M^{\prime}$ have a common non-zero submodule, say $N$. However, since $M$ and $M^{\prime}$ are prime they have the same support as $N$. Thus $Z=Z^{\prime}$.

To show that the map $Z \mapsto E$ is surjective, suppose that $E$ is an indecomposable injective. Because $X$ has the descending chain condition on closed subspaces, $E$ has a prime submodule and hence a prime critical noetherian submodule, say $M$. Hence $E$ is the image of the support of $M$.

The next result gives another class of spaces which have enough closed subspaces. It applies, for example, to the category of comodules over a coalgebra that is defined over a field. 
Proposition 4.8. Every weakly closed subspace of a locally finite space is closed.

Proof. Let $X$ be a locally finite space; that is, every $X$-module is a direct limit of finite length $X$-modules. Let $W$ be a weakly closed subspace of $X$. It suffices to show that $\operatorname{Mod} W$ is closed under products, because then the inclusion $i_{*}: \operatorname{Mod} W \rightarrow$ $\operatorname{Mod} X$ will have a left adjoint.

Let $\left\{M_{i} \mid i \in I\right\}$ be a collection of $W$-modules. By hypothesis, $\prod_{i \in I} M_{i}$, their product in $\operatorname{Mod} X$, is a sum of finite length $X$-modules. Let $N$ be a finite length submodule of $\prod_{i \in I} M_{i}$. For each $j \in I$ let $\rho_{j}: N \rightarrow M_{j}$ be the composition of the inclusion $N \rightarrow \prod_{i \in I} M_{i}$ with the projection $\prod_{i \in I} M_{i} \rightarrow M_{j}$. Write $K_{j}=\operatorname{ker} \rho_{j}$. Since $\bigcap_{j \in I} K_{j}$ is the kernel of the inclusion $N \rightarrow \prod_{i \in I} M_{i}$, it is zero; since $N$ has finite length, there is a finite subset $J \subset I$ such that $\bigcap_{j \in J} K_{j}=0$. It follows that the composition

$$
N \rightarrow \prod_{i \in I} M_{i} \rightarrow \prod_{j \in J} M_{j}
$$

is monic. Since $J$ is finite, $\prod_{j \in J} M_{j}$ is isomorphic to $\bigoplus_{j \in J} M_{j}$, and therefore belongs to $\operatorname{Mod} W$. Hence $N$ belongs to $\operatorname{Mod} W$.

We have shown that every finite length submodule of $\prod_{i \in I} M_{i}$ belongs to $\operatorname{Mod} W$. Since $\operatorname{Mod} W$ is closed under direct limits, we conclude that $\prod_{i \in I} M_{i}$ belongs to $\operatorname{Mod} W$.

\section{Closed points}

Notation. If $D$ is a division ring, we write $\operatorname{Spec} D$ for the space $\operatorname{Mod} D$ of right $D$-modules.

Definition 5.1. A closed point of a space $X$ is a closed subspace that is isomorphic to Spec $D$ for some division ring $D$. We call it a $D$-rational point of $X$.

We denote a closed point by a single letter, say $p$, and often write $p \in X$ to indicate that $p$ is a closed point of $X$. Since $\operatorname{Mod} p$ is equivalent to the category of modules over a division ring, it contains a unique simple module up to isomorphism; and because $\operatorname{Mod} p$ is closed under subquotients in $\operatorname{Mod} X$, that simple $p$-module is also simple as an $X$-module. We denote any module in the isomorphism class of this simple module by $\mathcal{O}_{p}$. Thus $D$ is isomorphic to $\operatorname{End}_{X} \mathcal{O}_{p}$, and every $p$-module is isomorphic to a direct sum of copies of $\mathcal{O}_{p}$.

Although a closed point determines a simple module, Proposition 5.7 shows that a simple module need not determine a closed point.

We will say that an $X$-module $M$ is compact if $\operatorname{Hom}_{X}(M,-)$ commutes with direct sums. If $X$ is locally noetherian, this is equivalent to the condition that $\operatorname{Hom}_{X}(M,-)$ commute with direct limits, and to the condition that $M$ be noetherian [19, Section 5.8].

Definition 5.2. An $X$-module $S$ is tiny if $\operatorname{Hom}_{X}(M, S)$ is a finitely generated



Lemma 5.3. Let $S$ be a tiny simple $X$-module. Then every compact submodule of a direct product of copies of $S$ is isomorphic to a finite direct sum of copies of $S$.

Proof. Let $D=\operatorname{End}_{X} S$. Let $P$ be a direct product of copies of $S$, and $M$ a compact submodule of $P$. Define $M_{0}=M$ and, if $M_{i-1}$ is non-zero, define $M_{i}$ inductively as follows. Since $M_{i-1} \neq 0$, there is projection $P \rightarrow S$ which does not 
vanish on $M_{i-1}$. Let $\rho_{i}: M \rightarrow S$ be the restriction of that projection and define $M_{i}=M_{i-1} \cap \operatorname{ker} \rho_{i}$. Since $\operatorname{dim}_{D} \operatorname{Hom}_{X}(M, S)<\infty$, there is a smallest integer $n$ such that $\rho_{n+1}=\sum_{i=1}^{n} \mu_{i} \rho_{i}$ for some $\mu_{i} \in D$. Therefore $\operatorname{ker} \rho_{n+1}$ contains $\operatorname{ker} \rho_{1} \cap \cdots \cap \operatorname{ker} \rho_{n}$. It follows that $0=M_{n}=\operatorname{ker} \rho_{1} \cap \cdots \cap \operatorname{ker} \rho_{n}$. Hence the map $\rho_{1} \oplus \ldots \oplus \rho_{n}: M \rightarrow S^{\oplus n}$ is an isomorphism.

Notation. If $S$ is a simple $X$-module, we write Sum $S$ for the full subcategory consisting of all modules that are isomorphic to a direct sum of copies of $S$. If $D$ denotes the endomorphism ring of $S$, then $\operatorname{Sum} S$ is equivalent to $\operatorname{Mod} D$.

In a Grothendieck category a sum of simple modules is isomorphic to a direct sum of simple modules. This is not true for $(\operatorname{Mod} \mathbb{Z})^{\mathrm{op}}$.

Lemma 5.4. Let $S$ be a simple $X$-module. The inclusion $i_{*}:$ SumS $\rightarrow \operatorname{Mod} X$ has a right adjoint $i^{!}$given by

$$
i^{!} N:=\text { the sum of all submodules of } N \text { that are isomorphic to } S \text {. }
$$

Proof. If $f: M \rightarrow N$ is a homomorphism of $X$-modules, then $f\left(i^{!} M\right) \subset i^{!} N$, so $i^{!}$ can be defined on morphisms by sending a morphism to its restriction. Thus $i^{!}$is a functor; it takes values in Sum $S$ because a sum of simple modules is isomorphic to a direct sum of simple modules. It is clear that $\operatorname{Hom}_{X}(S, M)=\operatorname{Hom}_{X}\left(S, i^{!} M\right)$, so $i^{!}$is right adjoint to $i_{*}$.

Alternatively, since Sum $S$ is closed under subquotients, $i_{*}$ is exact, and since Sum $S$ is closed under direct sums, $i_{*}$ commutes with direct sums, whence $i_{*}$ has a right adjoint.

Theorem 5.5. Suppose that $X$ is locally noetherian. Let $S$ be a simple $X$-module. The following are equivalent:

1. there is a closed point $p \in X$ such that $S \cong \mathcal{O}_{p}$;

2. the inclusion $i_{*}:$ Sum $S \rightarrow \operatorname{ModX}$ has a left adjoint;

3. every direct product of copies of $S$ is isomorphic to a direct sum of copies of $S$;

4. $S$ is tiny.

Proof. (1) $\Rightarrow$ (2) Since Mod $p$ consists of all direct sums of copies of $\mathcal{O}_{p}$, $\operatorname{Mod} p=$ Sum $S$. Hence the hypothesis that $p$ is closed ensures that $i_{*}$ has a left adjoint.

$(2) \Rightarrow(1)$ By Lemma $\left[5.4, i_{*}\right.$ has a right adjoint, so the hypothesis ensures that Sum $S$ satisfies the requirements to be the module category of a closed point.

$(2) \Leftrightarrow(3)$ Since $i_{*}$ is exact, it has a left adjoint if and only if it commutes with products; that is, if and only if $\operatorname{Sum} S$ is closed under products. But this is equivalent to condition (3).

(3) $\Rightarrow$ (4) If $M$ is compact, then

$$
\operatorname{Hom}_{X}(M, S)=\operatorname{Hom}_{X}\left(M, i_{*} S\right) \cong \operatorname{Hom}_{X}\left(i^{*} M, S\right) .
$$

But $i^{*} M$ is a quotient of $M$, so it is also compact. But $i^{*} M$ is in $\operatorname{Sum} S$, so it is a finite direct sum of copies of $S$. Thus $\operatorname{Hom}_{X}(M, S)$ is a finite direct sum of copies of $\operatorname{End}_{X} S$.

$(4) \Rightarrow(3)$ Let $P$ be a product of copies of $S$. Since $X$ is locally noetherian, $P$ is a direct limit of noetherian submodules. Each of those submodules is compact, and hence semisimple by Lemma 5.3. Therefore $P$ is semisimple. 
A locally noetherian space $X$ over a field $k$ is Ext-finite if $\operatorname{dim}_{k} \operatorname{Ext}_{X}^{i}(M, N)<\infty$ for all noetherian $X$-modules $M$ and $N$ and all integers $i$. If $A$ is a connected graded $k$-algebra such that $\operatorname{dim}_{k} A_{n}<\infty$ for all $n$, then [3, Section 3] gives conditions which ensure that the projective space with homogeneous coordinate ring $A$ is Ext-finite.

Corollary 5.6. Let $X$ be a locally noetherian space over $\operatorname{Spec} k$. If $X$ is Ext-finite, then every simple $X$-module is isomorphic to $\mathcal{O}_{p}$ for some closed point $p$. If $k$ is also algebraically closed, then $p$ is $k$-rational.

Proof. Let $S$ be a simple $X$-module. Then $\operatorname{Hom}_{X}(M, S)$ is finite dimensional over $k$ whenever $M$ is noetherian, so $S$ is tiny. Hence there is a closed point $p \in X$ such that $\mathcal{O}_{p} \cong S$. Since $X$ is Ext-finite, $\operatorname{Hom}_{X}\left(\mathcal{O}_{p}, \mathcal{O}_{p}\right)$ is a finite dimensional division algebra over $k$, and therefore equal to $k$ if $k$ is algebraically closed.

The following is routine, but we record it for later reference.

Proposition 5.7. Let $X$ be an affine space with coordinate ring $R$. The correspondences

$$
p \longleftrightarrow \mathcal{O}_{p} \longleftrightarrow \operatorname{Ann} \mathcal{O}_{p}
$$

set up a bijection between

1. closed points in $X$;

2. simple $R$-modules $S$ having finite length over $\operatorname{End}_{R} S$;

3. maximal two-sided ideals $\mathfrak{m}$ such that $R / \mathfrak{m}$ is artinian.

If $R$ is a countably generated algebra over an uncountable algebraically closed field $k$, then all closed points are $k$-rational, and they are in bijection with the finite dimensional simple $R$-modules.

The hypotheses on $k$ and $R$ in the last part of the proposition are needed because it is an open problem whether there exists a division algebra $D$ over an algebraically closed field $k$ such that $D \neq k$ but $D$ is finitely generated as a $k$-algebra. If there were such a $D$, then $\operatorname{Mod} D$ would have a single closed point, but that point would not be $k$-rational and would not correspond to a simple module of finite dimension over $k$.

The next result says that point modules over a reasonable graded algebra yield closed points in the associated projective space.

Proposition 5.8. Let $A$ be a right noetherian locally finite $\mathbb{N}$-graded $k$-algebra. Let $X$ be the projective space with homogeneous coordinate ring $A$. Let $M$ be an infinite dimensional graded right $A$-module such that $\operatorname{dim}_{k} M / N<\infty$ for all nonzero graded submodules $N \subset M$. If A satisfies the condition $\chi_{1}$ in [3, Section 3], then $\pi M \cong \mathcal{O}_{p}$ for some closed point $p \in X$.

Proof. The $\chi_{1}$ hypothesis implies that $\operatorname{Hom}_{X}\left(\mathcal{O}_{X}, \pi M(n)\right)$ has finite dimension for all $n \in \mathbb{Z}$. The hypothesis on $M / N$ ensures that $\pi M$ is a simple $X$-module. If $\mathcal{N}$ is any right noetherian $X$-module, then there is an epimorphism from a finite direct sum of copies of $\mathcal{O}_{X}(n)$ for various integers $n$ to $\mathcal{N}$. Hence $\operatorname{Hom}_{X}(\mathcal{N}, \pi M)$ is a subspace of a finite direct sum of various $\operatorname{Hom}_{X}\left(\mathcal{O}_{X}(n), \pi M\right)$, so finite dimensional. Thus $\pi M$ is a tiny simple module, and the result now follows from Theorem 5.5

We have the following consequences of Proposition 3.3. If $W$ is a weakly closed subspace of $X$, and $p$ is a closed point of $X$ such that $\mathcal{O}_{p}$ is a $W$-module, then $p$ is a closed point of $W$. If $Z$ is a closed subspace of $X$ and $p$ is a closed point of $Z$, 
then $p$ is a closed point of $X$. However, a closed point of a weakly closed subspace of $X$ need not be a closed point of $X$ : for example, if $M$ is an infinite dimensional simple module over a $\operatorname{ring} R$ such that $R / \operatorname{Ann} M$ is not artinian, then $M$ does not correspond to a closed point of $\operatorname{Mod} R$ even though $\operatorname{Sum} M$ is a closed point in Sum $M$.

Remark. Closed points do not always behave like zero-dimensional geometric objects. The following example has been emphasized by Artin and Zhang (private conversations).

Fix an algebraically closed field $k$. Let $E$ be an elliptic curve over $\operatorname{Spec} k, \sigma$ an automorphism of $E$ having infinite order, and $\mathcal{L}$ a line bundle of degree $\geq 3$ on $E$. Let $B=B(E, \sigma, \mathcal{L})$ be the twisted homogenous coordinate ring associated to this data 1. Then $B$ is a connected graded $k$-algebra of Gelfand-Kirillov dimension two, so $A:=B \otimes_{k} B$ is the homogeneous coordinate ring of a non-commutative projective space $X$ that is like a non-commutative 3-fold. If $\mathcal{F}$ and $\mathcal{G}$ are noetherian $X$-modules, then $\operatorname{Ext}_{X}^{i}(\mathcal{F}, \mathcal{G})$ is finite dimensional for all $i$. The $\operatorname{ring} A$ is Artin-Schelter-Gorenstein, so $X$ satisfies Serre duality. We use the $B$ - $B$-bimodule structure on $B$ to view it as a graded right $A$-module. Applying the quotient functor $\pi: \operatorname{GrMod} A \rightarrow \operatorname{Tails} A=\operatorname{Mod} X$ to $B$ produces an $X$-module $\mathcal{M}$. Since $\sigma$ has infinite order, every non-zero two-sided graded ideal in $B$ has finite codimension [1]; hence $\mathcal{M}$ is a simple $X$-module. By Corollary 5.6 there is a closed $k$-rational point $p \in X$ such that $\mathcal{M} \cong \mathcal{O}_{p}$. Because the Gelfand-Kirillov dimension of $B$ is two, and $A$ is Cohen-Macaulay with respect to GK-dimension, $\underline{\operatorname{Ext}}_{A}^{2}(B, A) \neq 0$. By 3 Theorem 8.1(5)], $\underline{\operatorname{Ext}}_{X}^{2}\left(\mathcal{O}_{p}, \mathcal{O}_{X}\right) \neq 0$. Serre duality now gives $H^{1}\left(X, \mathcal{O}_{p}(i)\right) \neq 0$ for some integer $i$. The non-vanishing of $H^{1}\left(X, \mathcal{O}_{p}(i)\right)$ indicates that the closed point $p$ behaves like a geometric object of dimension greater than zero.

Another example where a closed point behaves like a higher dimensional geometric object is given in [12]. There is a smooth non-commutative projective surface $X$ over $\mathbb{C}$ satisfying Serre duality, and a closed $\mathbb{C}$-rational point $p \in X$ such that $\operatorname{Ext}_{X}^{1}\left(\mathcal{O}_{p}, \mathcal{O}_{p}\right)=0$ and $\operatorname{Ext}_{X}^{2}\left(\mathcal{O}_{p}, \mathcal{O}_{p}\right)=k$. Thus $p$ behaves like a -2 -curve on $X$. This analogy is strengthened by the fact that $X$ is constructed from the sheaf of differential operators on $\mathbb{P}^{1}$ (and the projectivized cotangent bundle to $\mathbb{P}^{1}$ is a surface with a -2-curve). An alternative construction of $X$ is to take a projective compactification (as described before Proposition 8.11) of the affine space with coordinate ring a non-simple primitive factor ring of the enveloping algebra $U\left(\mathfrak{s l}_{2}\right)$; the associated graded ring of this factor is the coordinate ring of a singular quadric surface in $\mathbb{A}^{3}$, and the exceptional fiber in its minimal resolution is a -2-curve. All this suggests that $p$ behaves both like a point and like a -2-curve. Van den Bergh suggested that one should perhaps think of $X$ as its own blowup at $p$. The plausibility of this idea is reinforced by the fact that the ideal annihilating the simple $\mathfrak{s l}_{2}$-module corresponding to $p$ is idempotent (because $\mathfrak{s l}_{2}$ is semisimple), so the Rees ring construction of the blowup would reproduce the original surface.

Definition 5.9. Let $f: Z \rightarrow X$ be a weak map of spaces. Let $W$ be a weakly closed or weakly open subspace of $Z$ and let $p$ be a closed point of $X$. We say that the image of $W$ is $p$, and write $f(W)=p$, if $0 \neq f_{*}(\operatorname{Mod} W) \subset \operatorname{Mod} p$.

In that case there is a weak map $g: W \rightarrow p$ such that $i g=f \alpha$, where $\alpha: W \rightarrow Z$ and $i: p \rightarrow X$ are the inclusions; its direct image functor is $g_{*}=i^{*} f_{*} \alpha_{*}$. 
The map $f: \operatorname{Spec} k \rightarrow X$ induced by the inclusion

$$
\left(\begin{array}{ll}
k & k \\
0 & k
\end{array}\right) \rightarrow\left(\begin{array}{ll}
k & k \\
k & k
\end{array}\right)
$$

does not send the closed point of Spec $k$ to a closed point of $X$. The problem is that $f_{*}\left(\mathcal{O}_{\text {Spec } k}\right)$ is not a direct sum of copies of a single simple $X$-module. Similarly, if $f: \operatorname{Spec} k \rightarrow \operatorname{Spec}(k \times k)$ is the map induced by the inclusion of the diagonal matrices in $M_{2}(k), f$ does not send the closed point of Spec $k$ to a closed point of $\operatorname{Spec}(k \times k)$.

The bimodule $o_{p}$. Let $i: p \rightarrow X$ be the inclusion of a $D$-rational closed point of $X$. Following [25, Chapter 3], $p$ determines an $X$-X-bimodule $o_{p}$ defined by

$\mathcal{H}_{\mathrm{om}}\left(o_{p},-\right)=i_{*} i^{!}=$the sum of all submodules that are isomorphic to $\mathcal{O}_{p}$.

A left adjoint to $\mathcal{H o m}_{X}\left(o_{p},-\right)$ is $-\otimes_{X} o_{p}=i_{*} i^{*}$. If $\alpha: \operatorname{Spec} D \rightarrow p$ denotes the isomorphism, we may assume that $\alpha^{*}=\alpha^{!}$. By definition, $\operatorname{Hom}_{X}\left(\mathcal{O}_{p},-\right)=\alpha^{!} i^{!}$. It is natural to denote the left adjoint of $\operatorname{Hom}_{X}\left(\mathcal{O}_{p},-\right)$ by $-\otimes_{D} \mathcal{O}_{p}$. We write

$$
(-)^{*}=\operatorname{Hom}_{D}(-, D): \operatorname{Mod} D^{\mathrm{op}} \rightarrow \operatorname{Mod} D .
$$

The next result extends [25, Lemma 5.5.1].

Lemma 5.10. Let $p$ be a closed D-rational point of $X$. For all $j \geq 0$,

1. $\mathcal{E} x t_{X}^{j}\left(o_{p},-\right) \cong \operatorname{Ext}_{X}^{j}\left(\mathcal{O}_{p},-\right) \otimes_{D} \mathcal{O}_{p}$, and

2. $\mathcal{T}_{\text {or }}^{X}{ }_{j}^{X}\left(-, o_{p}\right) \cong \operatorname{Ext}_{X}^{j}\left(-, \mathcal{O}_{p}\right)^{*} \otimes_{D} \mathcal{O}_{p}$.

Proof. (1) Using the exactness of some of the functors, we have

$$
\begin{aligned}
\operatorname{Ext}_{X}^{j}\left(\mathcal{O}_{p},-\right) \otimes_{D} \mathcal{O}_{p} & =\left(i_{*} \alpha_{*}\right) \circ R^{j}\left(\alpha^{!} i^{!}\right) \\
& \cong R^{j}\left(i_{*} \alpha_{*} \alpha^{!} i^{!}\right) \\
& \cong R^{j}\left(i_{*} i^{!}\right) \\
& =\mathcal{E} x t_{X}^{j}\left(o_{p},-\right) .
\end{aligned}
$$

(2) We have

$$
M \otimes_{X} o_{p}=i_{*} i^{*} M=i_{*} \alpha_{*} \alpha^{*} i^{*} M=\left(\alpha^{*} i^{*} M\right) \otimes_{D} \mathcal{O}_{p},
$$

and

$$
\begin{aligned}
\alpha^{*} i^{*} M & \cong \operatorname{Hom}_{D}\left(\operatorname{Hom}_{D}\left(\alpha^{*} i^{*} M, D\right), D\right) \\
& \cong \operatorname{Hom}_{D}\left(\operatorname{Hom}_{X}\left(M, i_{*} \alpha_{*} D\right), D\right) \\
& =\operatorname{Hom}_{X}\left(M, \mathcal{O}_{p}\right)^{*} .
\end{aligned}
$$

Combining these two computations shows that

$$
M \otimes_{X} o_{p} \cong \operatorname{Hom}_{X}\left(M, \mathcal{O}_{p}\right)^{*} \otimes_{D} \mathcal{O}_{p} .
$$

Taking left derived functors of both sides, and using the fact that $(-)^{*}$ and $-\otimes_{D} \mathcal{O}_{p}$ are exact, gives (2).

If $p$ and $q$ are distinct closed points, then $o_{p} \otimes_{X} o_{q}=0$. 


\section{The COMPlement TO A WEAKLy ClOSED SUBSPACE}

Throughout this section $X$ is a locally noetherian space.

Weakly closed and weakly open subspaces were defined in Definitions 2.4 and 2.5. We begin this section by defining the complement to a weakly closed subspace. We will show that the complement to a weakly closed subspace is weakly open, and that every weakly open subspace arises as such a complement.

Definition 6.1. Let $X$ be a locally noetherian space, and let $W$ be a weakly closed subspace. The category of $X$-modules supported on $W$, which we denote by

$$
\operatorname{Mod}_{W} X
$$

is the full subcategory consisting of those $X$-modules which are the directed union of a family of submodules $M$ each of which has a finite filtration by submodules $0=M_{0} \subset M_{1} \subset \ldots \subset M_{r}=M$ such that each $M_{i} / M_{i-1}$ is a $W$-module. We call the modules $M_{i} / M_{i-1}$ the slices of the filtration.

Proposition 6.2 ([6 Prop. 8, p. 377]). Let $W$ be a weakly closed subspace of a locally noetherian space $X$. Then $\operatorname{Mod}_{W} X$ is a localizing subcategory of ModX.

Definition 6.3. The saturation of a weakly closed subspace $W$ of $X$ is the weakly closed subspace $W_{\text {sat }}$ defined by $\operatorname{Mod} W_{\text {sat }}=\operatorname{Mod}_{W} X$. We say that $W$ is saturated if $W=W_{\text {sat }}$.

Remarks. 1. It is obvious that $\left(W_{\text {sat }}\right)_{\text {sat }}=W_{\text {sat }}$.

2. The proof of Lemma 6.10 shows that if $W$ and $Z$ are weakly closed subspaces of a locally noetherian space $X$, then $Z_{\text {sat }} \cap W_{\text {sat }}=(Z \cap W)_{\text {sat }}$. This implies that $\left(Z \cap W_{\text {sat }}\right)_{\text {sat }}=(Z \cap W)_{\text {sat }}$.

Definition 6.4. If $W$ is a weakly closed subspace of a locally noetherian space $X$, its complement, denoted $X \backslash W$, is the non-commutative space defined by

$$
\operatorname{Mod} X \backslash W:=\operatorname{Mod} X / \operatorname{Mod}_{W} X
$$

We write $j: X \backslash W \rightarrow X$ for the map consisting of the adjoint pair of functors $\left(j^{*}, j_{*}\right)$ where $j^{*}: \operatorname{Mod} X \rightarrow \operatorname{Mod} X \backslash W$ is the quotient functor, and $j_{*}$ is its right adjoint (which exists because $\operatorname{Mod}_{W} X$ is a localizing subcategory).

One sees at once that $X \backslash X=\phi$ and $X \backslash \phi=X$.

Because $j_{*}$ is fully faithful, we have the following result.

Proposition 6.5. Let $W$ be a weakly closed subspace of a locally noetherian space $X$. Then $j: X \backslash W \rightarrow X$ is a weakly open immersion.

We will identify $\operatorname{Mod} X \backslash W$ with the full subcategory of $\operatorname{Mod} X$ consisting of those $X$-modules $M$ for which the canonical map $M \rightarrow j_{*} j^{*} M$ is an isomorphism. Hence we can speak of $X \backslash W$ as a weakly open subspace of $X$.

Since $\operatorname{Mod}_{W} X$ is a localizing subcategory of $\operatorname{Mod} X$, its inclusion has a right adjoint $\tau: \operatorname{Mod} X \rightarrow \operatorname{Mod}_{W} X ; \tau$ sends an $X$-module to its largest submodule that is supported on $W$. We sometimes call $\tau$ the $W$-torsion functor. By [6], for every $X$-module $M$ there is an exact sequence

$$
0 \rightarrow \tau M \rightarrow M \rightarrow j_{*} j^{*} M \rightarrow R^{1} \tau M \rightarrow 0,
$$

and $\tau j_{*} j^{*}=0$. 
Proposition 6.6. Every weakly open subspace of $X$ is of the form $X \backslash W$ for some weakly closed subspace $W$.

Proof. Let $j: U \rightarrow X$ be the inclusion of a weakly open subspace. By definition, $\operatorname{Mod} U$ is a full subcategory of $\operatorname{Mod} X$, and the inclusion $j_{*}: \operatorname{Mod} U \rightarrow \operatorname{Mod} X$ has an exact left adjoint $j^{*}$. Define

$$
\mathrm{T}:=\left\{M \in \operatorname{Mod} X \mid j^{*} M=0\right\} .
$$

Because $j^{*}$ is exact, $\mathrm{T}$ is closed under subquotients. Because $j^{*}$ has a right adjoint, $\mathrm{T}$ is closed under direct limits, whence the inclusion $\mathrm{T} \rightarrow \operatorname{Mod} X$ has a right adjoint. Hence $\mathrm{T}$ is the module category, $\operatorname{Mod} W$, for a weakly closed subspace $W$ of $X$.

Because $j_{*}$ is fully faithful, [19, Theorem 4.9, p. 180] shows that $\operatorname{Mod} U$ is equivalent to the quotient category $\operatorname{Mod} X / \mathrm{T}$. However, $\operatorname{Mod}_{W} X=\mathrm{T}$, so this quotient category is $\operatorname{Mod} X \backslash W$. Hence $U=X \backslash W$.

If $Z$ is a closed subspace of $X$, we call $X \backslash Z$ an open subspace of $X$.

The following two results are used in section 7

Lemma 6.7. Let $W$ be a weakly closed subspace of $X$ and let $U=X \backslash W$. Let $\alpha: W \rightarrow X$ and $j: U \rightarrow X$ be the inclusions. Then $\operatorname{Ext}_{X}^{1}\left(\alpha_{*} M, j_{*} N\right)=0$ for all $M \in \operatorname{Mod} W$ and all $N \in \operatorname{Mod} U$.

Proof. This result is a restatement of condition (b) in [6, Lemme 1, p. 370]; condition (c) of that result holds because $j^{*} j_{*} \cong \mathrm{id}_{U}$ and $j^{*}$ is a left adjoint to $j_{*}$.

Proposition 6.8. Let $U$ be a weakly open subspace of a locally noetherian space $X$. Let $j: U \rightarrow X$ denote the inclusion. Then $j_{*}$ commutes with direct limits.

Proof. This is a restatement of [6, Cor. 1, p. 379].

Definition 6.9. Let $U_{i}, i \in I$, be a small set of weakly open subspaces of $X$. Let $j_{i}: U_{i} \rightarrow X$ be the inclusion maps. We define their union, $\bigcup_{i \in I} U_{i}$, to be the weakly open subspace of $X$ whose module category is $\operatorname{Mod} X / \mathrm{T}$, where $\mathrm{T}$ is the localizing subcategory consisting of the $X$-modules $M$ such that $j_{i}^{*} M=0$ for all $i$. We say that the $U_{i}$ provide a weakly open cover of $X$ if their union is $X$; i.e., if $\mathrm{T}=0$. This is equivalent to the requirement that the diagonal $\operatorname{Mod} X \rightarrow \prod_{i} \operatorname{Mod} U_{i}$ is faithful. If each $U_{i}$ is open, we say that they provide an open cover of $X$.

The binary operation of union on weakly open subspaces is idempotent, commutative, and associative. The associativity of this operation allows one to define the union of a finite collection of weakly open subspaces by induction, and this notion of union agrees with that in Definition 6.9. If $U \subset V$ are weakly open subspaces of $X$, then $U \cup V=V$; so, for example, $U \cup \phi=U$ and $U \cup X=X$.

Lemma 6.10. Let $W$ and $Z$ be weakly closed subspaces of a locally noetherian space $X$. If $U=X \backslash W$ and $V=X \backslash Z$, then $U \cup V=X \backslash(W \cap Z)$.

Proof. By definition, $\operatorname{Mod} U \cup V$ is the quotient of $\operatorname{Mod} X$ by the localizing subcate$\operatorname{gory}^{\operatorname{Mod}_{W}} X \cap \operatorname{Mod}_{Z} X$, so it suffices to show that $\operatorname{Mod}_{W} X \cap \operatorname{Mod}_{Z} X=\operatorname{Mod}_{W \cap Z} X$. It suffices to prove that the full subcategories of noetherian modules are the same. It is obvious that $\bmod _{W \cap Z} X \subset \bmod _{W} X \cap \bmod _{Z} X$.

To prove the reverse inclusion, suppose that $M$ is in $\bmod _{W} X \cap \bmod _{Z} X$. There are finite filtrations $0=L_{0} \subset L_{1} \subset \ldots \subset L_{r}=M$ and $0=N_{0} \subset N_{1} \subset \ldots \subset N_{s}=M$ such that $L_{i} / L_{i-1} \in \bmod W$ and $N_{j} / N_{j-1} \in \bmod Z$ for all $i$ and $j$. Taking a common 
refinement of these filtrations produces a finite filtration of $M$ all of whose slices belong to $\bmod (W \cap Z)$, thus showing that $M \in \bmod _{W \cap Z} X$.

Remarks. 1. It is straightforward to show that if $S \subset T \subset A$ are Serre subcategories of a Grothendieck category $A$, then $T / S$ is a Serre subcategory of $A / S$, and $A / T$ is equivalent to $(\mathrm{A} / \mathrm{S}) /(\mathrm{T} / \mathrm{S})$ [19, Ex. 6, p. 174].

This has the following geometric interpretation: if $W \subset Z \subset X$ are weakly closed subspaces of $X$, then $X \backslash Z$ is a weakly open subspace of $X \backslash W$ (cf. Corollary 7.4). Example [6.11] shows that $X \backslash Z$ is not necessarily the complement to $Z \backslash W$ in $X \backslash W$; indeed, the last phrase does not always make sense, because $Z \backslash W$ need not be a weakly closed subspace of $X \backslash W$.

2. It follows from Lemma 6.10 and the previous remark that $U$ and $V$ are weakly open subspaces of $U \cup V$. It is easy to see that $U \cup V$ is the smallest weakly open subspace of $X$ that contains both $U$ and $V$.

Example 6.11. There is a space $X$ with closed subspaces $W \subset Z \subset X$ for which there is no weak map from $Z \backslash W$ to $X \backslash W$ making the following diagram commute:

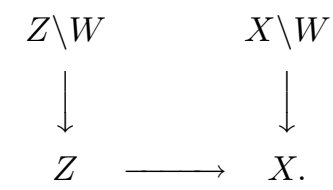

Let $k$ be a field, and let $X=\operatorname{Mod} R$ be the affine space with coordinate ring

$$
R=\left(\begin{array}{cc}
k & k \\
0 & k
\end{array}\right) .
$$

Let $p$ and $q$ be the closed points of $X$ corresponding to the simple right modules $\mathcal{O}_{p}$ and $\mathcal{O}_{q}$, labelled so that $\mathcal{O}_{p}$ is the projective simple module. Let $Z=\operatorname{Mod} R / N$, where $N$ is the ideal of strictly upper triangular matrices, and let $W=q$. Clearly $\operatorname{Mod} Z \cong \operatorname{Mod} k^{\oplus 2}$. Let $j: X \backslash W \rightarrow X$ be the inclusion. There is a non-split exact sequence $0 \rightarrow \mathcal{O}_{p} \rightarrow j_{*} j^{*} \mathcal{O}_{p} \rightarrow \mathcal{O}_{q} \rightarrow 0$. As full subcategories of $\operatorname{Mod} X, \operatorname{Mod} Z$ consists of all direct sums of copies of $\mathcal{O}_{p}$ and $\mathcal{O}_{q}, \operatorname{Mod} Z \backslash W$ consists of all direct sums of copies of $\mathcal{O}_{p}$, and $\operatorname{Mod} X \backslash W$ consists of all direct sums of copies of the indecomposable module $j_{*} j^{*} \mathcal{O}_{p}$. The truth of the claim is now apparent.

Example 6.11 is typical of what can happen in non-commutative geometry. Any space having a non-split extension of two non-isomorphic tiny simple modules has a closed subspace isomorphic to the space $X$ in Example 6.11; for example, the affine space with coordinate ring the enveloping algebra of any non-abelian solvable Lie algebra has such a closed subspace. The behavior in Example 6.11 is due to the fact that $\operatorname{Ext}_{X}^{1}\left(\mathcal{O}_{q}, \mathcal{O}_{p}\right) \neq 0$ even though $p$ and $q$ are distinct closed points. Although $Z$ consists of two closed points and $W$ is one of those points, $\operatorname{Mod} Z \cap \operatorname{Mod} X \backslash W=\phi$.

Lemma 6.12. Let $j: U \rightarrow X$ be the inclusion of a weakly open subspace. Then

1. every weakly open subspace of $U$ is a weakly open subspace of $X$;

2. if $i: V \rightarrow X$ is the inclusion of another weakly open subspace and ModV is contained in ModU, then there is a weakly open immersion $\beta: V \rightarrow U$ such that $i=j \beta$.

Proof. (1) Let $\beta: V \rightarrow U$ be the inclusion of a weakly open subspace of $U$. The functor $i_{*}:=j_{*} \beta_{*}$ is fully faithful, so we can view $\operatorname{Mod} V$ as a full subcategory of $\operatorname{Mod} X$. And $i_{*}$ has an exact left adjoint $\beta^{*} j^{*}$, so $V$ is weakly open in $X$. 
(2) Let $\beta_{*}: \operatorname{Mod} V \rightarrow \operatorname{Mod} U$ be the inclusion. Clearly $i_{*}=j_{*} \beta_{*}$. Since $j^{*}$ and $i^{*}$ have left adjoints, $\operatorname{Mod} U$ and $\operatorname{Mod} V$ are closed under products in $\operatorname{Mod} X$. Therefore $\operatorname{Mod} V$ is closed under products in $\operatorname{Mod} U$. Hence $\beta_{*}$ commutes with products. Since $j^{*} j_{*} \cong \mathrm{id}{ }_{U}, \beta_{*} \cong j^{*} i_{*}$; hence $\beta_{*}$ is left exact. Therefore $\beta_{*}$ has a left adjoint, say $\beta^{*}$. Notice that $i^{*} \cong \beta^{*} j^{*}$. To see that $\beta^{*}$ is exact, suppose that $0 \rightarrow L \rightarrow M \rightarrow N \rightarrow 0$ is exact in $\operatorname{Mod} U$. By [6, Cor. 1, p. 368], there is an exact sequence $0 \rightarrow L^{\prime} \rightarrow M^{\prime} \rightarrow N^{\prime} \rightarrow 0$ in $\operatorname{Mod} X$ such that the first sequence is obtained by applying $j^{*}$ to the second. It follows that $\beta^{*}$ applied to the first sequence is isomorphic to $i^{*}$ applied to the second; since $i^{*}$ is exact, we deduce that $\beta^{*}$ is exact. Thus $\beta$ is the inclusion of an open subspace of $U$.

Lemma 6.13. Let $W$ and $Z$ be weakly closed subspaces of a locally noetherian space $X$. Then

$$
\operatorname{Mod}_{W \cup Z} X=\operatorname{Mod}_{W \cdot Z} X=\operatorname{Mod}_{Z} \bullet W X
$$

and this is the smallest localizing subcategory of ModX that contains ModW and ModZ.

Proof. Since $W \cup Z$ contains $W$ and $Z, \operatorname{Mod}_{W \cup Z} X$ contains $\operatorname{Mod} W$ and $\operatorname{Mod} Z$. Since $\operatorname{Mod}_{W \cup Z} X$ is closed under extensions, it also contains $\operatorname{Mod} W \bullet Z$ and $\operatorname{Mod} Z \bullet W$, and hence contains $\operatorname{Mod}_{W \bullet Z} X$ and $\operatorname{Mod}_{Z \bullet W} X$. However, since $W \cup Z$ is contained in $W \bullet Z$ and in $Z \bullet W, \operatorname{Mod}_{W \cup Z} X$ is contained in $\operatorname{Mod}_{W \bullet Z} X$ and $\operatorname{Mod}_{Z \bullet W} X$. This gives (6.3). Finally, any localizing category that contains $\operatorname{Mod} W$ and $\operatorname{Mod} Z$ must contain $\operatorname{Mod} W \bullet Z$ and therefore $\operatorname{Mod}_{W \bullet Z} X$, so $\operatorname{Mod}_{W \bullet Z} X$ is the smallest localizing subcategory that contains $\operatorname{Mod} W$ and $\operatorname{Mod} Z$.

Definition 6.14. Let $U$ and $V$ be weakly open subspaces of a locally noetherian space $X$. The intersection of $U$ and $V$ is

$$
U \cap V:=X \backslash(W \cup Z),
$$

where $W$ and $Z$ are weakly closed subspaces such that $U=X \backslash W$ and $V=X \backslash Z$.

Remarks. 1. The definition of $U \cap V$ does not depend on the choice of $W$ and $Z$. Although $U$ may be the complement of many different weakly closed subspaces $W, \operatorname{Mod}_{W} X$ depends only on $U$ because, if $j: U \rightarrow X$ is the inclusion, $\operatorname{Mod}_{W} X$ consists of the modules on which $j^{*}$ vanishes; by Lemma $6.13 \operatorname{Mod}_{W \cup Z} X$ is the smallest localizing category containing $\operatorname{Mod}_{W} X$ and $\operatorname{Mod}_{Z} X$.

2. The remark before Example 6.11 implies that $U \cap V$ is a weakly open subspace of both $U$ and $V$. Proposition 6.15 shows that $U \cap V$ is the largest weakly open subspace of $X$ that is contained in both $U$ and $V$.

3. By Lemma 6.13, if $W$ and $Z$ are weakly closed subspaces of $X$, and $U=X \backslash W$ and $V=X \backslash Z$ are their complements, then

$$
U \cap V=X \backslash(W \bullet Z)=X \backslash(Z \bullet W) .
$$

4. If $W$ and $Z$ are closed subspaces of $X$, so is $Z \bullet W$ [25. Prop. 3.4.5]. Hence, if $U$ and $V$ are open subspaces of $X$, so is $U \cap V$.

Proposition 6.15. Let $U$ and $V$ be weakly open subspaces of a locally noetherian space $X$. Then Mod $\cap \cap V=\operatorname{Mod} U \cap \operatorname{Mod} V$.

Proof. Let $W$ and $Z$ be weakly closed subspaces such that $U=X \backslash W$ and $V=$ $X \backslash Z$. Let $i: U \rightarrow X, j: V \rightarrow X$, and $\alpha: U \cap V \rightarrow X$ be the inclusions. To make sense of the statement of the proposition, we identify Mod $U$ with the full 
subcategory of $\operatorname{Mod} X$ consisting of those modules $M$ for which the natural map $M \rightarrow i_{*} i^{*} M$ is an isomorphism, etc.

The quotient functor $\alpha^{*}: \operatorname{Mod} X \rightarrow \operatorname{Mod} U \cap V$ vanishes on $\operatorname{Mod}_{W} X$, so $\alpha^{*}$ factors through $i^{*}: \operatorname{Mod} X \rightarrow \operatorname{Mod} U$. Hence $\operatorname{Mod} U \cap V \subset \operatorname{Mod} U \cap \operatorname{Mod} V$.

It remains to show that if $M$ is in $\operatorname{Mod} U$ and $\operatorname{Mod} V$, then it is in $\operatorname{Mod} U \cap V$. Let $\tau: \operatorname{Mod} X \rightarrow \operatorname{Mod}_{W \cup Z} X \rightarrow \operatorname{Mod} X$ be the torsion functor. We must show that the middle arrow in the exact sequence

$$
0 \rightarrow \tau M \rightarrow M \rightarrow \alpha_{*} \alpha^{*} M \rightarrow R^{1} \tau M \rightarrow 0
$$

is an isomorphism. If $\tau M$ is non-zero, then $M$ has a non-zero submodule belonging to $\operatorname{Mod} W \cup Z$. By (3.4), $M$ therefore has a non-zero submodule belonging to either $\operatorname{Mod} W$ or $\operatorname{Mod} Z$. But $M \cong i_{*} i^{*} M \cong j_{*} j^{*} M$, so it cannot have such a non-zero submodule; we conclude that $\tau M=0$. If $R^{1} \tau M \neq 0$, then there is an essential extension $0 \rightarrow M \rightarrow M^{\prime} \rightarrow T \rightarrow 0$ with $T \in \operatorname{Mod} W \cup Z$. By (3.4), $T$ has a non-zero submodule belonging to either $\operatorname{Mod} W \operatorname{or} \operatorname{Mod} Z$, say $T^{\prime}$, and this would give an essential extension $0 \rightarrow M \rightarrow M^{\prime \prime} \rightarrow T^{\prime} \rightarrow 0$, contradicting the fact that $M \cong i_{*} i^{*} M \cong j_{*} j^{*} M$. Thus $R^{1} \tau M=0$ also, and the result follows.

Remarks. 1. Proposition 6.15 implies that $U \cap V$ is the largest weakly open subspace of $X$ contained in both $U$ and $V$.

2. The binary operation $\cap$ on weakly open subspaces is idempotent, commutative, and associative. The associativity allows us to define the intersection of a finite number of weakly open subspaces by induction. Both $\phi$ and $X$ are open subspaces of $X$, and if $U$ is weakly open, then $U \cap \phi=\phi$ and $U \cap X=X$. We show next that the lattice of weakly open subspaces is distributive.

Lemma 6.16. Let $U, V_{1}$, and $V_{2}$ be weakly open subspaces of a locally noetherian space $X$. Then

$$
U \cap\left(V_{1} \cup V_{2}\right)=\left(U \cap V_{1}\right) \cup\left(U \cap V_{2}\right) .
$$

Proof. Let $W, Z_{1}$, and $Z_{2}$ be weakly closed subspaces of $X$ such that $U=X \backslash W$ and $V_{i}=X \backslash Z_{i}$. Then $V_{1} \cup V_{2}=X \backslash\left(Z_{1} \cap Z_{2}\right)$, and $U \cap\left(V_{1} \cup V_{2}\right)=X \backslash\left(W \bullet\left(Z_{1} \cap Z_{2}\right)\right)$ by (6.4). On the other hand $\left(U \cap V_{1}\right) \cup\left(U \cap V_{2}\right)=X \backslash\left(W \bullet Z_{1} \cap W \bullet Z_{2}\right)$. To prove the result it suffices to show that $\operatorname{Mod}_{W \bullet Z_{1} \cap W \bullet Z_{2}} X=\operatorname{Mod}_{W \bullet\left(Z_{1} \cap Z_{2}\right)} X$. Since $W \bullet\left(Z_{1} \cap Z_{2}\right) \subset W \bullet Z_{1} \cap W \bullet Z_{2}$, one inclusion is obvious. To prove the other, it suffices to show that $\operatorname{Mod}\left(W \bullet Z_{1} \cap W \bullet Z_{2}\right)$ is contained in $\operatorname{Mod}_{W \bullet\left(Z_{1} \cap Z_{2}\right)} X$.

Let $M \in \operatorname{Mod}\left(W \bullet Z_{1} \cap W \bullet Z_{2}\right)$. There are exact sequences $0 \rightarrow L_{i} \rightarrow M \rightarrow$ $N_{i} \rightarrow 0, i=1,2$, with $L_{i} \in \operatorname{Mod} Z_{i}$ and $N_{1}, N_{2} \in \operatorname{Mod} W$. The slices in the filtration $0 \subset L_{1} \cap L_{2} \subset L_{1} \subset M$ belong to $\operatorname{Mod} Z_{1} \cap Z_{2}, \operatorname{Mod} Z_{1} \cap W$, and Mod $W$ respectively, all of which are subcategories of $\operatorname{Mod} W \bullet\left(Z_{1} \cap Z_{2}\right)$, so $M \in \operatorname{Mod}_{W \bullet\left(Z_{1} \cap Z_{2}\right)} X$. This completes the proof.

Definition 6.17. We say that a weakly closed subspace $W$ of $X$ contains a weakly open subspace $U$ of $X$ if $\operatorname{Mod} U \subset \operatorname{Mod} W$; more precisely, if $\alpha: W \rightarrow X$ and $j: U \rightarrow$ $X$ are the inclusion maps, we say that $W$ contains $U$ if $j_{*}(\operatorname{Mod} U) \subset \alpha_{*}(\operatorname{Mod} W)$.

Lemma 6.18. Let $U$ and $Z$ be respectively a weakly open subspace and a weakly closed subspace of a locally noetherian space $X$. Let $j: U \rightarrow X$ and $\delta: Z \rightarrow X$ denote the inclusion maps. Then $U$ is contained in $Z$ if and only if there is a weak map $\nu: U \rightarrow Z$ such that $\delta \nu=j$. If that is the case, then

1. $U$ is a weakly open subspace of $Z$; 
2. if $U=X \backslash W$, then $U=Z \backslash(Z \cap W)$;

3. $U$ is an open subspace of $Z$ if it is an open subspace of $X$.

Proof. If $U$ is contained in $Z$, then $\operatorname{Mod} U \subset \operatorname{Mod} Z$; so, if $\nu_{*}: \operatorname{Mod} U \rightarrow \operatorname{Mod} Z$ is the inclusion, $\delta_{*} \nu_{*}=j_{*}$. Hence $\nu_{*}$ defines a weak map $\nu: U \rightarrow Z$ such that $\delta \nu=j$. Conversely, if there is a weak map $\nu: U \rightarrow Z$ such that $\delta \nu=j$, then $\delta_{*} \nu_{*} \cong j_{*}$, so $\delta^{!} j_{*} \cong \delta^{!} \delta_{*} \nu_{*} \cong \nu_{*}$ and $\delta_{*} \delta^{!} j_{*} \cong \delta_{*} \nu_{*} \cong j_{*}$, whence $j_{*}(\operatorname{Mod} U) \subset \delta_{*}(\operatorname{Mod} Z)$; in other words, $\operatorname{Mod} U$ is contained in $\operatorname{Mod} Z$.

(1) Because $\operatorname{Mod} U$ is a full subcategory of $\operatorname{Mod} X$, it is also a full subcategory of $\operatorname{Mod} Z$. Because $\nu^{*}:=j^{*} \delta_{*}$ is an exact left adjoint to $\delta^{!} j_{*} \cong \nu_{*}, U$ is a weakly open subspace of $Z$.

(2) Since $\nu: U \rightarrow Z$ is a weakly open immersion, $\operatorname{Mod} U=\operatorname{Mod} Z / \mathrm{T}$, where $\mathrm{T}$ is the full subcategory of $\operatorname{Mod} Z$ where $\nu^{*}$ vanishes; however, if $M \in \operatorname{Mod} Z$, then $\nu^{*} M=0$ if and only if $j^{*} M=0$, so $\mathrm{T}=\operatorname{Mod} Z \cap \operatorname{Mod}_{W} Z$. Therefore $U=Z \backslash\left(Z \cap W_{\text {sat }}\right)$. However, by the remark after Definition 6.3, $Z \backslash\left(Z \cap W_{\text {sat }}\right)=$ $Z \backslash(Z \cap W)$, so $U=Z \backslash(Z \cap W)$.

(3) If $U$ is open in $X$, then $U=X \backslash W$ for some closed subspace $W$ of $X$. Proposition 3.3 shows that $Z \cap W$ is then a closed subspace of $Z$, so $Z \backslash(Z \cap W)$ is an open subspace of $Z$.

Definition 6.19. The weak closure of a weakly open subspace $U$ of $X$ is the smallest weakly closed subspace of $X$ that contains $U$; we denote it by $\bar{U}$. This makes sense because an intersection of weakly closed subspaces is weakly closed - see the remarks after Proposition 3.3

If $j: U \rightarrow X$ is the inclusion of a weakly open subspace of a locally noetherian space $X$, then $\operatorname{Mod} \bar{U}$ consists of all $X$-modules $N$ for which there exist a $U$-module $P$ and $X$-submodules $L \subset M \subset j_{*} P$ such that $N \cong M / L$. It is clear that this subcategory of $\operatorname{Mod} X$ contains $\operatorname{Mod} U$, is closed under subquotients, and is closed under direct sums because direct sums are exact in $\operatorname{Mod} X$ and $j_{*}$ commutes with direct sums.

Lemma 6.20. If $U$ is a weakly open subspace of a locally noetherian space $X$, then

1. $U \cap(X \backslash \bar{U})=\phi$;

2. if $U \cap V \neq \phi$ for all non-empty weakly open subspaces $V$ in $X$, then $(\bar{U})_{\text {sat }}=$ $X$.

Proof. (1) Let $W$ be a saturated weakly closed subspace of $X$ such that $U=X \backslash W$, and write $j: U \rightarrow X$ for the inclusion map. Let $\tau: \operatorname{Mod} X \rightarrow \operatorname{Mod}_{W} X=\operatorname{Mod} W$ be the $W$-torsion functor. Let $M \in \operatorname{Mod} X$. Since $M / \tau M$ embeds in $j_{*} j^{*} M$, it is a $\bar{U}$-module. Hence $M \in \operatorname{Mod}(\bar{U} \bullet W)$, and we conclude that $\bar{U} \bullet W=X$. Therefore $\phi=X \backslash(\bar{U} \bullet W)=X \backslash(\bar{U} \cup W)=(X \backslash \bar{U}) \cap(X \backslash W)$, as claimed.

(2) The hypothesis implies that $X \backslash \bar{U}=\phi$, whence $(\bar{U})_{\text {sat }}=X$.

If $X$ is the affine space in Example 6.11 then $U=X \backslash q$ is an open subspace such that $\bar{U}=X$, but $U \cap(X \backslash p)=U \cap q=\phi$. It would be worthwhile to find conditions which ensure that $U \cap V \neq \phi$ for all non-empty weakly open subspaces $V$ when $\bar{U}=X$.

There is not a useful analogue of quasi-compactness for weakly open covers: if $W_{i}$ is the weakly closed subspace of Spec $\mathbb{Z}$ defined at the beginning of section 3 and $U_{i}$ is its weakly open complement, then $\operatorname{Spec} \mathbb{Z}$ is the union of the $U_{i}$ but is not the union of any finite subset of these. 
However, the same proof as for the commutative case shows that affine spaces satisfy an analogue of quasi-compactness for open covers.

Lemma 6.21. If $\left\{U_{i} \mid i \in I\right\}$ is an open cover of an affine space $X$, then there is a finite subset $F \subset I$ such that $\left\{U_{i} \mid i \in F\right\}$ is an open cover for $X$.

Proof. Suppose that $R$ is a coordinate ring for $X$, and that $Z_{i}, i \in I$, are closed subspaces of $X$ such that $U_{i}=X \backslash Z_{i}$. Let $K_{i}$ be the two-sided ideal of $R$ corresponding to $Z_{i}$; that is, $\operatorname{Mod} Z_{i}=\operatorname{Mod} R / K_{i}$. Let $K$ denote the sum of all the $K_{i}$. If $j_{i}: U_{i} \rightarrow X$ is the inclusion, then $j_{i}^{*}\left(R / K_{i}\right)=0$, so $j_{i}^{*}(R / K)=0$ for all $i \in I$. Hence $R / K=0$, and it follows that $R$ is contained in $\sum_{i \in F} K_{i}$ for some finite set $F \subset I$.

Now we show that the $U_{i}, i \in F$, provide an open cover of $X$. If $M$ is a non-zero module such that $j_{i}^{*} M=0$ for all $i \in F$, then $j_{i}^{*} N=0$ for all $i \in F$ for every simple subquotient $N$ of $M$. It follows that $N \in \operatorname{Mod} Z_{i}$ for all $i \in F$, so $N K_{i}=0$ for all $i \in F$; this contradicts the fact that $R=\sum_{i \in F} K_{i}$.

\section{Containment And intersection of SUbspaces}

We have defined containment, intersection, and union for a pair of weakly closed subspaces and for a pair of weakly open subspaces. We would also like to define containment and intersection for pairs of subspaces where one of the subspaces is weakly open and the other is weakly closed.

We begin with the following question: if $Z$ and $W$ are weakly closed subspaces of $X$ such that $W \cap Z=\phi$, is $Z$ a weakly closed subspace of $X \backslash W$ ? Unfortunately, it need not be.

To determine exactly when $Z$ is a weakly closed subspace of $X \backslash W$ we must address the following general question. If $\beta: Z \rightarrow X$ is a weak map, and $j$ : $X \backslash W \rightarrow X$ is the inclusion of a weakly open subspace, when is there a weak map $\gamma: Z \rightarrow X \backslash W$ such that the diagram

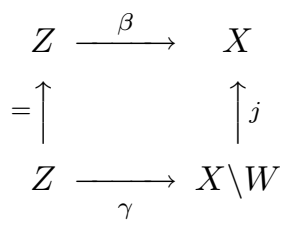

commutes? The case when $Z$ is a weakly closed subspace of $X$ such that $Z \cap W=\phi$ is relevant to the question posed in the previous paragraph.

Proposition 7.1. Let $\beta: Z \rightarrow X$ be a weak map. Let $W$ be a weakly closed subspace of $X$; let $\alpha: W \rightarrow X$ and $j: X \backslash W \rightarrow X$ be the inclusion maps. The following are equivalent:

1. $\operatorname{Hom}_{X}\left(\alpha_{*} M, \beta_{*} N\right)=\operatorname{Ext}_{X}^{1}\left(\alpha_{*} M, \beta_{*} N\right)=0$ for all $M \in \operatorname{Mod} W$ and all $N \in$ $\operatorname{Mod} Z$;

2. $j_{*} j^{*} \beta_{*} \cong \beta_{*}$;

3. there is a unique weak map $\gamma: Z \rightarrow X \backslash W$ such that $\beta=j \gamma$.

Suppose that these conditions hold. Then

1. if $\beta$ is a map, so is $\gamma$;

2. if $\beta$ is an affine map, so is $\gamma$;

3. if $\beta$ is a weakly closed (resp. weakly open, closed) immersion, so is $\gamma$. 
Proof. First we establish the uniqueness claim in part (3). If $\gamma$ and $\gamma^{\prime}$ are weak maps from $Z$ to $X \backslash W$ such that $\beta=j \gamma=j \gamma^{\prime}$, then $\beta_{*} \cong j_{*} \gamma_{*} \cong j_{*} \gamma_{*}^{\prime}$. But $j^{*} j_{*} \cong \mathrm{id}$, so $j^{*} \beta_{*} \cong \gamma_{*} \cong \gamma_{*}^{\prime}$. Because $\gamma_{*}$ and $\gamma_{*}^{\prime}$ are naturally equivalent, the maps $\gamma$ and $\gamma^{\prime}$ are the same (Definition [2.3).

Define $\gamma_{*}: \operatorname{Mod} Z \rightarrow \operatorname{Mod} X \backslash W$ by $\gamma_{*}=j^{*} \beta_{*}$. Since $\gamma_{*}$ is exact, it defines a weak map $\gamma: Z \rightarrow X \backslash W$. (It is not necessarily true that $j \gamma=\beta$.) Let $\tau: \operatorname{Mod} X \rightarrow$ $\operatorname{Mod}_{W} X$ denote the torsion functor that takes the $W$-support of a module. If $N \in \operatorname{Mod} Z$, then there is an exact sequence

$$
0 \rightarrow \tau\left(\beta_{*} N\right) \rightarrow \beta_{*} N \rightarrow j_{*} j^{*}\left(\beta_{*} N\right) \rightarrow R^{1} \tau\left(\beta_{*} N\right) \rightarrow 0
$$

$(1) \Rightarrow(2)$ The vanishing of Hom implies that $\beta_{*} N$ cannot have a non-zero $W$ submodule, so $\tau\left(\beta_{*} N\right)=0$. The vanishing of $\operatorname{Ext}^{1}$ implies that $R^{1} \tau\left(\beta_{*} N\right)=0$. Hence the natural transformation $\beta_{*} \rightarrow j_{*} j^{*} \beta_{*}$ is an isomorphism.

(2) $\Rightarrow(3)$ Since $j_{*} \gamma_{*}=j_{*} j^{*} \beta_{*} \cong \beta_{*}, j \gamma=\beta$.

(3) $\Rightarrow$ (1) If there is a weak map $\gamma: Z \rightarrow X \backslash W$ such that $j \gamma=\beta$, then $\beta_{*} \cong j_{*} \gamma_{*}$, so $j^{*} \beta_{*} \cong j^{*} j_{*} \gamma_{*} \cong \gamma_{*}$, so we may assume that $\gamma_{*}=j^{*} \beta_{*}$. It follows that $j_{*} j^{*} \beta_{*} \cong j_{*} \gamma_{*} \cong \beta_{*}$, whence (2) holds. But $j_{*} j^{*} \beta_{*} N$ is the largest essential extension of $\beta_{*} N$ by a module in $\operatorname{Mod}_{W} X$. It follows that one cannot extend $\beta_{*} N$ in an essential way by a $W$-module. In other words, if $0 \rightarrow \beta_{*} N \rightarrow E^{0} \rightarrow E^{1} \rightarrow \ldots$ is a minimal injective resolution in $\operatorname{Mod} X$, then $\operatorname{Hom}_{X}\left(\alpha_{*} M, E^{1}\right)=0$ for all $W$ modules $M$; (1) follows.

Now suppose that the three equivalent conditions hold.

(1) Suppose that $\beta$ is a map. Thus $\beta_{*}$ has a left adjoint $\beta^{*}$. If $L \in \operatorname{Mod} X \backslash W$ and $N \in \operatorname{Mod} Z$, then

$$
\begin{aligned}
\operatorname{Hom}_{Z}\left(\beta^{*} j_{*} L, N\right) & \cong \operatorname{Hom}_{X}\left(j_{*} L, \beta_{*} N\right) \\
& \cong \operatorname{Hom}_{X}\left(j_{*} L, j_{*} j^{*} \beta_{*} N\right) \\
& \cong \operatorname{Hom}_{X \backslash W}\left(L, j^{*} \beta_{*} N\right) \\
& \cong \operatorname{Hom}_{X \backslash W}\left(L, \gamma_{*} N\right) .
\end{aligned}
$$

Therefore $\gamma^{*}:=\beta^{*} j_{*}$ is a left adjoint to $\gamma_{*}$, so $\gamma$ is a map.

(2) Suppose that $\beta$ is an affine map. We have just seen that $\gamma$ is a map. Since $\beta_{*}$ is faithful and $\beta_{*} \cong j_{*} \gamma_{*}, \gamma_{*}$ is faithful. Since $\beta_{*}$ has a right adjoint, $\beta^{!}$say, $\gamma_{*}$ has a right adjoint, namely $\gamma^{!}:=\beta^{!} j_{*}$. Therefore $\gamma$ is an affine map.

(3) Suppose that $\beta$ is a weakly closed immersion. Then $\beta_{*}$ is fully faithful, $\beta_{*}(\operatorname{Mod} Z)$ is closed under subquotients in $\operatorname{Mod} X$, and $\beta_{*}$ has a right adjoint $\beta$ ! Thus $\gamma^{!}:=\beta^{!} j_{*}$ is right adjoint to $\gamma_{*}$. Since $\gamma^{!} \gamma_{*} \cong \beta^{!} j_{*} j^{*} \beta_{*} \cong \beta^{!} \beta_{*}$, we have

$$
\operatorname{Hom}_{X \backslash W}\left(\gamma_{*} L, \gamma_{*} N\right) \cong \operatorname{Hom}_{Z}\left(L, \gamma^{!} \gamma_{*} N\right) \cong \operatorname{Hom}_{Z}\left(L, \beta^{!} \beta_{*} N\right)=\operatorname{Hom}_{Z}(L, N) .
$$

Hence $\gamma_{*}$ is fully faithful, and we can view $\operatorname{Mod} Z$ as a full subcategory of $\operatorname{Mod} X \backslash W$ via $\gamma_{*}$. To see that $\gamma_{*}$ is a weakly closed immersion, we must check that $\gamma_{*}(\operatorname{Mod} Z)$ is closed under subquotients in $\operatorname{Mod} X \backslash W$.

Let $N$ be a $Z$-module and suppose that $0 \rightarrow K \rightarrow \gamma_{*} N \rightarrow L \rightarrow 0$ is an exact sequence in $\operatorname{Mod} X \backslash W$. Applying $j_{*}$ produces an exact sequence

$$
0 \rightarrow j_{*} K \rightarrow j_{*} j^{*} \beta_{*} N \cong \beta_{*} N \rightarrow j_{*} L \rightarrow R^{1} j_{*} K .
$$

Since $j_{*} K \rightarrow \beta_{*} N$ is an $X$-submodule of a module in $\beta_{*}(\operatorname{Mod} Z)$, which is closed under $X$-submodules, $j_{*} K \in \beta_{*}(\operatorname{Mod} Z)$; thus $j_{*} K \cong \beta_{*} \beta^{!} j_{*} K$. Hence $K \cong$ $j^{*} j_{*} K \cong j^{*} \beta_{*} \beta^{!} j_{*} K \cong \gamma_{*} \gamma^{!} K$. In particular, $K \in \gamma_{*}(\operatorname{Mod} Z)$. If we apply 
$\gamma_{*}$ to the exact sequence $0 \rightarrow \gamma^{!} K \rightarrow N \rightarrow N / \gamma^{!} K \rightarrow 0$, we obtain an exact sequence $0 \rightarrow \gamma_{*} \gamma^{!} K \cong K \rightarrow \gamma_{*} N \rightarrow \gamma_{*}\left(N / \gamma^{!} K\right) \rightarrow 0$. It follows that $L \cong \gamma_{*}\left(N / \gamma^{!} K\right) \in \gamma_{*}(\operatorname{Mod} Z)$. Hence $\gamma_{*}(\operatorname{Mod} Z)$ is closed under subquotients. This completes the proof that $\gamma$ is a weakly closed immersion if $\beta$ is.

It follows that $\gamma$ is a closed immersion if $\beta$ is.

Finally, suppose that $\beta$ is a weakly open immersion. Thus $\beta_{*}$ has an exact left adjoint $\beta^{*}$. Since $\beta$ is a map, so is $\gamma$. In other words, $\gamma_{*}$ has a left adjoint $\gamma^{*}:=\beta^{*} j_{*}$. If $M \in \operatorname{Mod} W$ and $N \in \operatorname{Mod} Z$, then

$$
\operatorname{Hom}_{Z}\left(\beta^{*} \alpha_{*} M, N\right) \cong \operatorname{Hom}_{X}\left(\alpha_{*} M, \beta_{*} N\right)=0,
$$

so $\beta^{*}$ vanishes on all $W$-modules. Since $\beta^{*}$ is exact and commutes with direct limits, it vanishes on $\operatorname{Mod}_{W} X$. By [6] Cor. 2 and 3, pp. 368-369], there is an exact functor $H: \operatorname{Mod} X \backslash W \rightarrow \operatorname{Mod} Z$ such that $H j^{*} \cong \beta^{*}$. However, $H \cong H j^{*} j_{*} \cong$ $\beta^{*} j_{*}=\gamma^{*}$. Therefore $\gamma_{*}$ has an exact left adjoint, thus showing that $\gamma$ is a weakly open immersion.

Definition 7.2. Let $W$ be a weakly closed subspace of $X$. Let $V$ be either a weakly closed or a weakly open subspace of $X$. We say that $V$ is contained in $X \backslash W$, and write $V \subset X \backslash W$, if $\operatorname{Mod} V \subset \operatorname{Mod} X \backslash W$.

Remarks. 1. If $\operatorname{Mod} V \subset \operatorname{Mod} X \backslash W$, then, by Lemma 6.7 $\operatorname{Ext}_{X}^{1}(M, N)=0$ for all $W$-modules $M$ and all $V$-modules $N$. Hence, by Proposition 7.1, the inclusion $\gamma_{*}: \operatorname{Mod} V \rightarrow \operatorname{Mod} X \backslash W$ is the direct image functor for a weak map $\gamma: V \rightarrow X \backslash W$ satisfying $\beta=j \gamma$. Furthermore, $V$ is a weakly closed (resp., weakly open, closed) subspace of $X \backslash W$ if it is weakly closed (resp., weakly open, closed) in $X$.

2. Let $Z$ be a weakly closed subspace of $X$. If $Z \subset X \backslash W$, then $Z \cap W=\phi$. However, if $Z \cap W=\phi$, then Proposition 7.1 says that $Z$ is contained in $X \backslash W$ if and only if $\operatorname{Ext}_{X}^{1}(M, N)=0$ for all $W$-modules $M$ and all $Z$-modules $N$.

3. Example 6.11 exhibits distinct closed points $p, q \in X$ such that $q$ is not contained in $X \backslash p$. Compare this with Corollaries 7.3 and 8.7

4. If $V$ is contained in $X \backslash W$, so are all weakly closed and all weakly open subspaces of $V$.

Corollary 7.3. Let $p$ be a closed point of $X$, and let $W$ be a weakly closed subspace of $X$ that does not contain $p$. Let $j: X \backslash W \rightarrow X$ be the inclusion. Suppose that $\operatorname{Ext}_{X}^{1}\left(j_{*} M, \mathcal{O}_{p}\right)=0$ for all $W$-modules $M$. Then

1. $p$ is contained in $X \backslash W$;

2. $p$ is a closed point of $X \backslash W$;

3. if $q$ is a closed point in $X \backslash W$, then $j_{*} \mathcal{O}_{q} \cong \mathcal{O}_{p}$ if and only if $q=p$.

Proof. Let $\beta: p \rightarrow X$ denote the inclusion. By Proposition 17.1, there is a closed immersion $\gamma: p \rightarrow X \backslash W$ such that $\beta=j \gamma$. Thus we can view $p$ as a closed subspace of $X \backslash W$. The fact that $p$ is a closed point of $X \backslash W$ follows from the fact that $\gamma_{*} \mathcal{O}_{p}=j^{*} \beta_{*} \mathcal{O}_{p}$ is simple in $\operatorname{Mod} X \backslash W$ (a localization functor sends a simple module to a simple module or zero). Part (3) follows from the fact that $j_{*}$ is fully faithful.

Corollary 7.4. Let $W \subset Z \subset X$ be weakly closed subspaces of $X$. Then $X \backslash Z$ is a weakly open subspace of $X \backslash W$. Precisely, there is a weakly open immersion 
$\gamma: X \backslash Z \rightarrow X \backslash W$ such that the following diagram commutes:

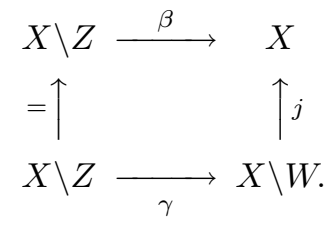

Proof. By Lemma [6.7 $\operatorname{Ext}_{X}^{1}\left(-, \beta_{*} N\right)$ vanishes on all $Z$-modules. Thus the first condition in Proposition 7.1 is satisfied, and it follows that such a $\gamma$ exists.

Remarks. 1. By Proposition 6.15 if $U$ and $V$ are weakly open subspaces of $X$, then there is a commutative diagram of weakly open immersions

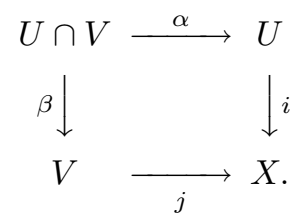

Corollary 7.4 also confirms this: let $W$ and $Z$ be weakly closed subspaces such that $U=X \backslash W$ and $V=X \backslash Z$; let $\gamma: U \cap V=X \backslash(W \cup Z) \rightarrow X$ be the inclusion; since $W \subset W \cup Z$, there is a weakly open immersion $\alpha: X \backslash(W \cup Z) \rightarrow X \backslash W$ such that $i \alpha=\gamma$ (by the proof of Proposition $7.1 \alpha$ is obtained by defining $\alpha_{*}=i^{*} \gamma_{*}$ ); similarly, there is a weakly open immersion $\beta: X \backslash(W \cup Z) \rightarrow X \backslash Z$ such that $j \beta=\gamma$.

2. In contrast to the case for schemes [10, Ch. II, Ex. 4.3], we cannot conclude that $U \cap V$ is affine even if $U$ and $V$ are open affine subspaces of an affine space $X$. This is not a surprise. Suppose that $X$ is affine with coordinate ring $R$. If $i$ and $j$ are affine maps, then $U$ and $V$ are affine, with coordinate rings $R_{U}$ and $R_{V}$ say; we can choose $R$ such that $i$ and $j$ are induced by ring homomorphisms $R \rightarrow R_{U}$ and $R \rightarrow R_{V}$, both of which are necessarily epimorphisms in the category of rings. However, when $R$ is not commutative, there may not be a ring structure on $R_{U} \otimes_{R} R_{V}$ such that the natural map $R \rightarrow R_{U} \otimes_{R} R_{V}$ is a ring homomorphism.

Proposition 7.5. Let $U$ and $V$ be weakly open subspaces of a locally noetherian space $X$. Then $U \cap V$ is the fiber product $U \times_{X} V$ of $U$ and $V$ in the category of spaces (with maps as the morphisms).

Proof. Consider the diagram (7.2) above. Suppose that $f: Y \rightarrow U$ and $g: Y \rightarrow V$ are maps such that if $=j g$. We must show that there is a unique map $h: Y \rightarrow U \cap V$ such that $f=\alpha h$ and $g=\beta h$.

The inclusion $\gamma: U \cap V \rightarrow X$ is given by $\gamma_{*}=i_{*} \alpha_{*}=j_{*} \beta_{*}$. Since $\gamma^{*} \cong \alpha^{*} i^{*}$, we have $\gamma^{*} i_{*} \cong \alpha^{*}$.

The image of $i_{*} f_{*}$ is the same as that of $j_{*} g_{*}$, so is contained in $\operatorname{Mod} U \cap \operatorname{Mod} V=$ $\operatorname{Mod} U \cap V$, whence $i_{*} f_{*} \cong \gamma_{*} \gamma^{*} i_{*} f_{*}$. Thus

$$
f_{*} \cong i^{*} i_{*} f_{*} \cong i^{*} \gamma_{*} \gamma^{*} i_{*} f_{*} \cong i^{*}\left(i_{*} \alpha_{*} \alpha^{*} i^{*}\right) i_{*} f_{*} \cong \alpha_{*} \alpha^{*} f_{*} .
$$

By Proposition 7.1, there is a unique map $h: Y \rightarrow U \cap V$ such that $f=\alpha h$. The proof of 7.1 shows that $h_{*}=\alpha^{*} f_{*}$. The "same" argument shows there is a unique map $h^{\prime}: Y \rightarrow U \cap V$ such that $g=\beta h^{\prime}$, and $h_{*}^{\prime}=\beta^{*} g_{*}$. Therefore

$$
h_{*}=\alpha^{*} f_{*} \cong \gamma^{*} i_{*} f_{*} \cong \gamma^{*} j_{*} g_{*} \cong \beta^{*} g_{*}=h_{*}^{\prime},
$$

and we conclude that $h=h^{\prime}$, completing the proof. 
We now generalize the question posed prior to Proposition 7.1. If $Z$ and $W$ are weakly closed subspaces of a space $X$, when is $Z \backslash(Z \cap W)$ a weakly closed subspace of $X \backslash W$ ? Example 6.11 shows that the answer is not "always".

Proposition 7.6. Let $W$ and $Z$ be weakly closed subspaces of a locally noetherian space $X$. Denote the inclusion maps by

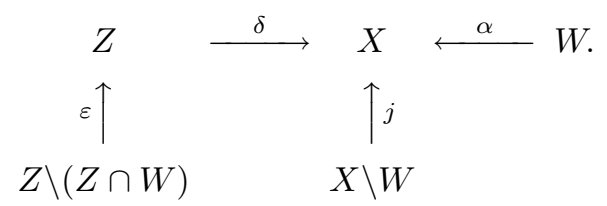

There is a weakly closed immersion $\gamma: Z \backslash(Z \cap W) \rightarrow X \backslash W$ such that $j \gamma=\delta \varepsilon$ if and only if $\operatorname{Ext}_{X}^{1}\left(\alpha_{*} M, \delta_{*} \varepsilon_{*} N\right)=0$ for all $M \in \operatorname{Mod} W$ and all $N \in \operatorname{Mod} Z \backslash(Z \cap W)$. In that case, if $\delta$ is a closed immersion, so is $\gamma$.

Proof. We set $\beta=\delta \varepsilon$. Thus $\beta: Z \backslash(Z \cap W) \rightarrow X$ is a weak map.

$(\Rightarrow)$ Suppose there is a weak map $\gamma$ such that $j \gamma=\delta \varepsilon$. By Proposition 7.1 $\operatorname{Ext}_{X}^{1}\left(\alpha_{*} M, \delta_{*} \varepsilon_{*} N\right)=0$ for all $M$ and $N$.

$(\Leftarrow)$ Proposition 7.1 immediately yields a weak map $\gamma: Z \backslash(Z \cap W) \rightarrow X \backslash W$ such that $j \gamma=\beta=\delta \varepsilon$. Explicitly, $\gamma_{*}=j^{*} \beta_{*}=j^{*} \delta_{*} \varepsilon_{*}$. Furthermore, $\beta_{*} \cong j_{*} j^{*} \beta_{*}$.

To show that $\gamma$ is a weakly closed immersion we show that $\gamma_{*}(\operatorname{Mod} Z \backslash(Z \cap W))$ is closed under subquotients in $\operatorname{Mod} X \backslash W$ and that $\gamma_{*}$ is exact, fully faithful, and has a right adjoint.

Since $\gamma_{*}$ is a composition of left exact functors, it is left exact. To show that $\gamma_{*}$ is exact, it suffices to show that $j^{*} \delta_{*}\left(R^{1} \varepsilon_{*}\right)=0$. Now $R^{1} \varepsilon_{*}$ takes values in $\operatorname{Mod}_{Z \cap W} Z$, and $j^{*} \delta_{*}$ vanishes on this because $j^{*}$ vanishes on $\operatorname{Mod}_{W} X$. Hence $j^{*} \delta_{*}\left(R^{1} \varepsilon_{*}\right)=0$, and $\gamma_{*}$ is exact.

Because $j^{*}$ and $\delta_{*}$ have right adjoints, they commute with direct limits. By Lemma 6.7 $\varepsilon_{*}$ commutes with direct limits. Therefore $\gamma_{*}$ commutes with direct limits. Since $\gamma_{*}$ is also exact, it has a right adjoint, which we denote by $\gamma^{\text {! }}$.

It is useful to have a precise formula for $\gamma^{!}$. Since $j^{*} \delta_{*}$ vanishes on $\operatorname{Mod}_{Z \cap W} Z$, $j^{*} \delta_{*}$ vanishes on the kernel and cokernel of the natural transformation $\operatorname{id}_{Z} \rightarrow \varepsilon_{*} \varepsilon^{*}$. Hence the natural transformation $j^{*} \delta_{*} \rightarrow j^{*} \delta_{*} \varepsilon_{*} \varepsilon^{*}$ is an isomorphism. That is, $j^{*} \delta_{*} \cong \gamma_{*} \varepsilon^{*}$. Taking right adjoints, $\delta^{!} j_{*} \cong \varepsilon_{*} \gamma^{!}$. It follows that

$$
\gamma^{!} \cong \varepsilon^{*} \delta^{!} j_{*}
$$

The fact that $\gamma_{*}$ is fully faithful follows from the calculation

$$
\begin{aligned}
\operatorname{Hom}_{Z \backslash(Z \cap W)}(L, N) & \cong \operatorname{Hom}_{Z \backslash(Z \cap W)}\left(\varepsilon^{*} \varepsilon_{*} L, N\right) \\
& \cong \operatorname{Hom}_{Z}\left(\varepsilon_{*} L, \varepsilon_{*} N\right) \\
& \cong \operatorname{Hom}_{Z}\left(\varepsilon_{*} L, \delta^{!} \delta_{*} \varepsilon_{*} N\right) \\
& \cong \operatorname{Hom}_{X}\left(\delta_{*} \varepsilon_{*} L, \beta_{*} N\right) \\
& \cong \operatorname{Hom}_{X}\left(\delta_{*} \varepsilon_{*} L, j_{*} j^{*} \beta_{*} N\right) \\
& \cong \operatorname{Hom}_{X \backslash W}\left(j^{*} \delta_{*} \varepsilon_{*} L, j^{*} \beta_{*} N\right) .
\end{aligned}
$$

Because $\gamma_{*}$ is fully faithful, the unit $\operatorname{id}_{Z \backslash(Z \cap W)} \rightarrow \gamma^{\prime} \gamma_{*}$ is an isomorphism.

Now we check that $\gamma_{*}(\operatorname{Mod} Z \backslash(Z \cap W))$ is closed under subquotients in $\operatorname{Mod} X \backslash W$. Let $N \in \operatorname{Mod} Z \backslash(Z \cap W)$, and consider an exact sequence $0 \rightarrow P \rightarrow \gamma_{*} N \rightarrow Q \rightarrow 0$ 
in $\operatorname{Mod} X \backslash W$. Hence $j_{*} P$ is an $X$-submodule of $j_{*} \gamma_{*} N \cong \delta_{*} \varepsilon_{*} N$. But $\delta_{*}(\operatorname{Mod} Z)$ is closed under submodules, so $j_{*} P$ is a $Z$-module. Formally, $j_{*} P \cong \delta_{*} \delta^{!} j_{*} P$, whence

$$
P \cong j^{*} j_{*} P \cong j^{*} \delta_{*} \delta^{!} j_{*} P \cong \gamma_{*} \varepsilon^{*} \delta^{!} j_{*} P \cong \gamma_{*} \gamma^{!} P .
$$

Thus $P$ is in the image of $\gamma_{*}$. Now, if we apply $\gamma_{*}$ to the exact sequence $0 \rightarrow \gamma^{!} P \rightarrow$ $\gamma^{!} \gamma_{*} N \cong N \rightarrow N / \gamma^{!} P \rightarrow 0$, we obtain the original sequence $0 \rightarrow \gamma_{*} \gamma^{!} P \cong P \rightarrow$ $\gamma_{*} N \rightarrow \gamma_{*}\left(N / \gamma^{!} P\right) \rightarrow 0$, from which it follows that $Q \cong \gamma_{*}\left(N / \gamma^{!} P\right)$.

Finally, we show that if $\delta$ is a closed immersion, so is $\gamma$. If $\delta^{*}$ is a left adjoint to $\delta_{*}$, then

$$
\begin{aligned}
\operatorname{Hom}_{Z}\left(\varepsilon^{*} \delta^{*} j_{*} P, L\right) & \cong \operatorname{Hom}_{X}\left(j_{*} P, \delta_{*} \varepsilon_{*} L\right) \cong \operatorname{Hom}_{X}\left(j_{*} P, j_{*} j^{*} \beta_{*} L\right) \\
& \cong \operatorname{Hom}_{X \backslash W}\left(j^{*} j_{*} P, j^{*} \beta_{*} L\right) \cong \operatorname{Hom}_{X \backslash W}\left(P, \gamma_{*} L\right) .
\end{aligned}
$$

Hence $\gamma^{*}:=\varepsilon^{*} \delta^{*} j_{*}$ is a left adjoint to $\gamma_{*}$, showing that $\gamma$ is a closed immersion.

Lemma 7.7. In the setting of Proposition 7.6.

$$
j_{*} \gamma_{*}(\operatorname{Mod} Z \backslash(Z \cap W))=\delta_{*}(\operatorname{Mod} Z) \cap j_{*}(\operatorname{Mod} X \backslash W) .
$$

Proof. Since $\delta_{*}$ and $j_{*}$ are fully faithful, the category on the right-hand side is a full subcategory of $\operatorname{Mod} X$. So is the category on the left-hand side, because $\gamma_{*}$ is fully faithful. Hence to verify this equality it is enough to check it on objects. Because $j_{*} \gamma_{*} \cong \delta_{*} \varepsilon_{*}$, the left-hand side is contained in the right-hand side. Conversely, a module on the right-hand side is of the form $\delta_{*} L$ for some $L \in \operatorname{Mod} Z$, and it satisfies $\delta_{*} L \cong j_{*} j^{*} \delta_{*} L$. The proof of Proposition 7.6 showed that $j^{*} \delta_{*} \cong \gamma_{*} \varepsilon^{*}$, so $\delta_{*} L \cong j_{*} j^{*} \delta_{*} L \cong j_{*} \gamma_{*} \varepsilon^{*} L$, which is in the left-hand side.

Definition 7.8. Let $W$ and $Z$ be weakly closed subspaces of a locally noetherian space $X$, and set $U=X \backslash W$. Let $\alpha: W \rightarrow X$ and $\beta: Z \backslash(Z \cap W) \rightarrow Z \rightarrow X$ be the inclusions. If $\operatorname{Ext}_{X}^{1}\left(\alpha_{*} M, \beta_{*} N\right)=0$ for all $M \in \operatorname{Mod} W$ and all $N$ in $\operatorname{Mod} Z \backslash(Z \cap W)$, we define $Z \cap U$ by declaring

$$
\operatorname{Mod} Z \cap U:=\operatorname{Mod} Z \cap \operatorname{Mod} U .
$$

Remarks. In these remarks, $W$ and $Z$ are weakly closed subspaces of $X, U$ denotes $X \backslash W$, and we use the notation of Proposition 7.6

1. Example 6.11 shows that $Z \cap U$ is not always defined. In that example, $Z \cap(X \backslash q)$ is not defined because $Z \backslash(Z \cap q)=p$ and $\operatorname{Ext}_{X}^{1}\left(\mathcal{O}_{q}, \mathcal{O}_{p}\right) \neq 0$. Therefore $Z \cap U$ need not be defined even if $U \rightarrow X$ is affine.

2. If $Z$ is contained in $U$ (Definition 7.2), then Lemma6.7 ensures that $Z \cap U$ is defined, and $Z \cap U=Z$.

3. If $U$ is contained in $Z$ (Definition 6.17), then $Z \backslash(Z \cap W)=U$ by Lemma 6.18, so Lemma 6.7 ensures that $Z \cap U$ is defined, and $Z \cap U=U$. In particular, $X \cap U=U$ and $\bar{U} \cap U=U$.

4. Lemma 6.7 implies that $W \cap U$ is defined, and $W \cap U=\emptyset$.

5. If $V \subset U$ is a weakly open subspace of $U$, then $V$ is weakly open in $X$, and $\bar{V} \subset \bar{U}$, where these are the weak closures in $X$. We do not know if $\bar{V} \cap U$ is defined.

6. If $U$ is also weakly closed in $X$, we can use Definition 3.2 to define $Z \cap U$; in that case $Z \cap U$ is a weakly closed subspace of $Z, U$, and $X$, and is also weakly open in $Z$ by Proposition 3.3 We claim that $Z \cap U$ is also defined according to Definition [7.8], comparing Definitions [3.2] and [7.8, it is then clear that the two definitions of $Z \cap U$ agree. To verify the claim we must show, in the notation of Proposition [7.6, that there is a map $\gamma: Z \backslash(Z \cap W) \rightarrow X \backslash W$ making the diagram 
(7.3) commute. By Proposition [7.1, it suffices to show that $j_{*} j^{*} \delta_{*} \varepsilon_{*} \cong \delta_{*} \varepsilon_{*}$. Since $j: U \rightarrow X$ is a weakly closed immersion, the natural map $\delta_{*} \varepsilon_{*} N \rightarrow j_{*} j^{*} \delta_{*} \varepsilon_{*} N$ is epic. The kernel is $\tau\left(\delta_{*} \varepsilon_{*} N\right)$, where $\tau$ is the $W$-torsion functor. However, zero is the only $Z \cap W$-submodule of $\varepsilon_{*} N$, so zero is the only $W$-submodule of $\delta_{*} \varepsilon_{*} N$, whence $\tau\left(\delta_{*} \varepsilon_{*} N\right)=0$.

7. If $Z$ is also weakly open in $X$, we can use Definition 6.14 to define $Z \cap$ $U$; we claim that $Z \cap U$ is also defined according to Definition 7.8 comparing Definition 7.8 with Proposition 6.15, it is then clear that the two definitions of $Z \cap U$ agree. To verify the claim we must show that $\operatorname{Ext}_{X}^{1}\left(\alpha_{*} M, \delta_{*} \varepsilon_{*} N\right)=0$ for all $M \in \operatorname{Mod} W$ and all $N \in \operatorname{Mod} Z \backslash(Z \cap W)$. Since $Z$ is both weakly open and weakly closed, $\delta_{*}$ is exact. Therefore the spectral sequence (2.2) for $\delta: Z \rightarrow X$ collapses, and gives $\operatorname{Ext}_{X}^{1}\left(\alpha_{*} M, \delta_{*} \varepsilon_{*} N\right) \cong \operatorname{Ext}_{Z}^{1}\left(\delta^{*} \alpha_{*} M, \varepsilon_{*} N\right)$. Since $\delta^{*} \alpha_{*} M$ is a $Z \cap W$-module, Lemma 6.7 applied to the open subspace $Z \backslash(Z \cap W)$ of $Z$ shows that $\operatorname{Ext}_{Z}^{1}\left(\delta^{*} \alpha_{*} M, \varepsilon_{*} N\right)=0$.

Proposition 7.9. Let $W$ and $Z$ be weakly closed subspaces of a locally noetherian space $X$, and set $U=X \backslash W$. Suppose that $Z \cap U$ is defined. Then

1. $Z \cap U=Z \backslash(Z \cap W)$;

2. $Z \cap U$ is a weakly open subspace of $Z$ and a weakly closed subspace of $U$;

3. if $Z$ is closed in $X$, then $Z \cap U$ is closed in $U$;

4. if $U \rightarrow X$ is an affine map, so is $Z \cap U \rightarrow Z$.

Proof. Part (1) follows from Lemma 7.7, and parts (2) and (3) follow from Proposition 7.6

(4) In the notation of Proposition 7.6 we must show that $\varepsilon_{*}$ is faithful and has a right adjoint, where $\varepsilon$ is the weakly open immersion $Z \cap U \rightarrow Z$. We already know that $\varepsilon_{*}$ is faithful and commutes with direct sums, since $\varepsilon^{*}$ is a localization. Since $j: U \rightarrow X$ is affine, $j_{*}$ and hence $j_{*} \gamma_{*}$ is exact. But $j_{*} \gamma_{*} \cong \delta_{*} \varepsilon_{*}$; since a sequence of $Z$-modules is exact if and only if it is exact when considered as a sequence of $X$-modules, it follows that $\varepsilon_{*}$ is exact.

The language we are using is close to that of topology. For this paragraph only we will say that the notions of weakly closed and weakly open subspaces provide a space with an almost-topological structure. If $Z$ and $U$ are respectively a weakly closed and weakly open subspace of $X$, it (almost) makes sense to ask if the almosttopological structures on $Z$ and $U$, which they have because they are spaces, are the same as the induced almost-topological structures on them. The next result shows this is true for $U$, but the example following it shows this is false for $Z$.

Proposition 7.10. Let $U$ be a weakly open subspace of a locally noetherian space $X$. Then every weakly closed subspace of $U$ is of the form $Z \cap U$ for some weakly closed subspace $Z$ of $X$.

Proof. Let $V$ be a weakly closed subspace of $U$, and let $\gamma: V \rightarrow U$ and $j: U \rightarrow X$ be the inclusions. We seek a weakly closed subspace $Z$ of $X$ such that $V=Z \cap U$. That is, if $\delta: Z \rightarrow X$ denotes the inclusion, we seek a commutative diagram

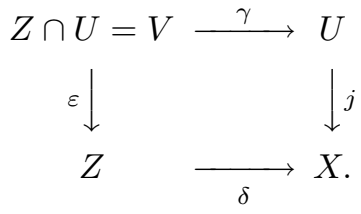


By hypothesis, $j_{*}$ and $\gamma_{*}$ are fully faithful, so we can think of them as inclusions of full subcategories.

Let A be the full subcategory of $\operatorname{Mod} X$ consisting of all $X$-modules $N$ that can be written as $N \cong M / L$, where $L \subset M \subset j_{*} \gamma_{*} P$ are $X$-submodules of $j_{*} \gamma_{*} P$ for some $P \in \operatorname{Mod} V$.

Clearly $\mathrm{A}$ is closed under subquotients in $\operatorname{Mod} X$. Hence the inclusion $\delta_{*}: \mathrm{A} \rightarrow$ $\operatorname{Mod} X$ is exact. If $N_{i}=M_{i} / L_{i}, i \in I$, belong to A, where $L_{i} \subset M_{i} \subset j_{*} \gamma_{*} P_{i}$, then, taking direct sums in $\operatorname{Mod} X$, we have $\bigoplus_{i} N_{i} \cong \bigoplus_{i} M_{i} / \bigoplus_{i} L_{i}$, and

$$
\bigoplus_{i} L_{i} \subset \bigoplus_{i} M_{i} \subset \bigoplus j_{*} \gamma_{*} P_{i} \cong j_{*} \gamma_{*}\left(\bigoplus_{i} P_{i}\right),
$$

where the final direct sum is taken in $\operatorname{Mod} V$ and the final isomorphism is due to the fact that $j_{*}$ and $\gamma_{*}$ commute with direct limits. It follows that $\bigoplus_{i} N_{i}$ is in A. Thus $\mathrm{A}$ is closed under direct limits in $\operatorname{Mod} X$. Hence $\delta_{*}$ commutes with direct limits, and therefore has a right adjoint $\delta^{!}$. So we can define a weakly closed subspace $Z$ of $X$ by declaring $\operatorname{Mod} Z$ to be $A$.

We now show that $j_{*} \gamma_{*}(\operatorname{Mod} V)=\delta_{*}(\operatorname{Mod} Z) \cap j_{*}(\operatorname{Mod} U)$. One inclusion is easy: if $P \in \operatorname{Mod} V$, then $j_{*} \gamma_{*} P$ is in $\operatorname{Mod} Z$ by definition, thus showing that the righthand side contains the left-hand side. Now suppose that $N$ is in the right-hand side. Thus $N=M / L$, where $L \subset M \subset j_{*} \gamma_{*} P$ for some $P \in \operatorname{Mod} V$. There are inclusions $j^{*} L \subset j^{*} M \subset j^{*} j_{*} \gamma_{*} P \cong \gamma_{*} P$. Since $\gamma_{*}(\operatorname{Mod} V)$ is closed under subquotients in $\operatorname{Mod} U, j^{*} M / j^{*} L$ is in $\gamma_{*}(\operatorname{Mod} V)$. Hence $j^{*} N \in \gamma_{*}(\operatorname{Mod} V)$. But $N$ is in $j_{*}(\operatorname{Mod} U)$, so $N \cong j_{*} j^{*} N \in j_{*} \gamma_{*}(\operatorname{Mod} V)$.

To show that $Z \cap U$ is defined we must show that $\operatorname{Ext}_{X}^{1}\left(\alpha_{*}-, \delta_{*} \varepsilon_{*}-\right)=0$. Since $\delta_{*} \varepsilon_{*}=j_{*} \delta_{*}$, this follows from Lemma 6.7. Hence $Z \cap U$ is defined, and it follows from the previous paragraph and Lemma 7.7 that $V=Z \cap U$.

If we call the subspace $Z$ constructed in the previous proof the weak closure of $V$ in $X$, and denote it by $\bar{V}$ (cf. Definition 6.19), then Proposition 7.10) says that $V=\bar{V} \cap U$ whenever $V$ is a weakly closed subspace of a weakly open subspace $U$. Thus $V$ is the analogue of a locally closed subscheme in the sense that it is a weakly open subspace of its weak closure $\bar{V}$.

If products are exact in $\operatorname{Mod} X$ (for example, if $X$ is affine), there is a version of Proposition 7.10 with "weakly closed" replaced by "closed". In that case, the equality $\bar{V} \cap U=V$ extends the result that if $R_{\mathcal{S}}$ is an Ore localization of a ring $R$, and $J$ is a right ideal of $R_{\mathcal{S}}$, then $J=(J \cap R) R_{\mathcal{S}}$.

Example 7.11. We exhibit a closed subspace $Z \subset X$ and an open subspace of $Z$ that is not of the form $Z \cap U$ for any weakly open subspace $U$ of $X$. We retain the notation in Example6.11. The weakly open subspaces of $X$ are

$$
\phi, X \backslash p=q, X \backslash q, X
$$

and the weakly open subspaces of $Z$ are

$$
\phi, Z \backslash p=q, Z \backslash q=p, Z .
$$

Therefore $p$, which is weakly open (even open) in $Z$, is not equal to $Z \cap U$ for any weakly open subspace $U$ in $X$. 


\section{EfFEctive Divisors}

Van den Bergh [25] has defined the notion of a divisor on a non-commutative space. The reader is referred there for the definition and basic results. Further properties of effective divisors can be found in [11.

Definition 8.1 ([11]). An effective divisor $Y$ on a non-commutative space $X$ is an invertible proper subobject $o_{X}(-Y)$ of $o_{X}$ in the category of $X$-X-bimodules.

If $X$ is a scheme such that $Q \operatorname{coh} X$ is a Grothendieck category, then there is a bijection between effective Cartier divisors and effective divisors in the sense of Definition 8.1 .

If $Y$ is an effective divisor, then $o_{X}(-Y)$ is a proper ideal in $o_{X}$, so the results in [25, Section 3.5] show that $Y$ determines, and is determined by, a non-empty closed subspace of $X$ which we also denote by $Y$. The category $\operatorname{Mod} Y$ is the full subcategory of $\operatorname{Mod} X$ consisting of those modules $M$ such that the natural map $M(-Y) \rightarrow M$ is zero.

We write $i: Y \rightarrow X$ for the inclusion of the effective divisor. The left and right adjoints to the inclusion $i_{*}: \operatorname{Mod} Y \rightarrow \operatorname{Mod} X$ are related via their derived functors as follows:

$$
R^{1} i^{!} \cong i^{*}(-)(Y) \quad \text { and } \quad L_{1} i^{*} \cong i^{!}(-)(-Y) .
$$

Because $i^{*}$ is right exact, $R^{2} i^{!}=0$. Therefore the spectral sequence (2.1) degenerates, giving a long exact sequence

$$
\begin{aligned}
0 & \rightarrow \operatorname{Ext}_{Y}^{1}\left(M, i^{!} N\right) \rightarrow \operatorname{Ext}_{X}^{1}\left(i_{*} M, N\right) \rightarrow \operatorname{Hom}_{Y}\left(M, R^{1} i^{!} N\right) \\
& \rightarrow \operatorname{Ext}_{Y}^{2}\left(M, i^{!} N\right) \rightarrow \operatorname{Ext}_{X}^{2}\left(i_{*} M, N\right) \rightarrow \operatorname{Ext}_{Y}^{1}\left(M, R^{1} i^{!} N\right) \rightarrow \cdots
\end{aligned}
$$

There is an exact sequence

$$
0 \longrightarrow o_{X}(-Y) \longrightarrow o_{X} \stackrel{\varphi}{\longrightarrow} o_{Y} \longrightarrow 0
$$

of bimodules, where $-\otimes_{X} o_{Y}=i_{*} i^{*}$ and $\mathcal{H}^{\circ} m_{X}\left(o_{Y},-\right)=i_{*} i^{!}$. Applying $M \otimes_{X}-$ to (8.3) produces an exact sequence

$$
0 \rightarrow \mathcal{T}_{1}^{X}\left(M, o_{Y}\right)=i^{!} M(-Y) \rightarrow M(-Y) \stackrel{\psi}{\longrightarrow} M \rightarrow i^{*} M \rightarrow 0 .
$$

We note that $\mathcal{T}_{\text {or }}^{X}\left(-, o_{Y}\right)=0$ for $r \geq 2$.

Proposition 8.2. Let $i: Y \rightarrow X$ and $\alpha: W \rightarrow X$ be the inclusions of an effective divisor and a weakly closed subspace respectively. Suppose that $M(Y)$ and $M(-Y)$ belong to ModW whenever $M$ does. Then $W \cap Y$ is an effective divisor on $W$ if and only if $W \cap Y \neq \phi$ and $\mathcal{T}_{\text {or }}^{X}\left(o_{Y}, o_{W}\right)=0$. The last condition is equivalent to $i^{*} \alpha_{*} \alpha^{!}$vanishing on all injective $X$-modules.

Proof. By Proposition 3.3 there is a commutative diagram

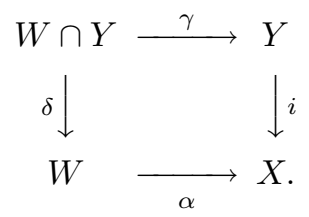

in which $W \cap Y$ is a closed subspace of $W$. Furthermore, $\gamma_{*} \delta^{*} \cong i^{*} \alpha_{*}$ and $\delta_{*} \gamma^{!} \cong$ $\alpha ! i_{*}$. 
Before proceeding, we establish the equivalence claimed in the last sentence of the proposition. By definition of $\mathcal{T}_{o r}{ }^{X}$, if $E$ is an injective $X$-module, then

$$
\mathcal{H o m}_{X}\left(\mathcal{T}_{o r}^{X}\left(o_{Y}, o_{W}\right), E\right)=\mathcal{E} x t_{X}^{1}\left(o_{Y}, \mathcal{H} \operatorname{Hom}_{X}\left(o_{W}, E\right)\right)=R^{1} i^{!}\left(\alpha_{*} \alpha^{!} E\right) .
$$

Therefore $\mathcal{T}_{o r}^{X}\left(o_{Y}, o_{W}\right)=0$ if and only if $i^{*} \alpha_{*} \alpha^{!} E=0$ for all injective $X$-modules E.

Now suppose that $W \cap Y$ is an effective divisor on $W$. Since an effective divisor is non-empty, $W \cap Y \neq \phi$. Furthermore, $\delta^{*} \cong R^{1} \delta^{!}(-)(W \cap Y)$, so $\delta^{*}$ vanishes on all injective $W$-modules. Since $\alpha^{!}$sends injectives to injectives, $\gamma_{*} \delta^{*} \alpha^{!}$vanishes on all injective $X$-modules. Hence $i^{*} \alpha_{*} \alpha^{!}$vanishes on all injective $X$-modules.

Conversely, suppose that $W \cap Y \neq \phi$ and that $\mathcal{T}_{\text {or }}^{X}\left(o_{Y}, o_{W}\right)=0$. Because $W \cap Y \neq \phi, o_{W \cap Y} \neq 0$. Hence to show that $W \cap Y$ is an effective divisor on $W$, we must prove the invertibility of the ideal $I$ of $o_{W}$ that is the kernel in the exact sequence $0 \rightarrow I \rightarrow o_{W} \rightarrow o_{W \cap Y} \rightarrow 0$ of $W$-W-bimodules. By definition, $\mathcal{H}_{o m}\left(o_{W \cap Y}, M\right)=\delta_{*} \delta^{!} M$ for all $M \in \operatorname{Mod} W$. The ideal $I$ is defined by the requirement that if $E$ is an injective $W$-module, then the sequence

$$
0 \rightarrow \delta_{*} \delta^{!} E \rightarrow E \rightarrow \mathcal{H o m}_{W}(I, E) \rightarrow 0
$$

is exact. Equivalently, $\mathcal{H o m}_{W}(I, E)$ is defined by the requirement that

$$
0 \rightarrow \alpha_{*} \delta_{*} \delta^{!} E \rightarrow \alpha_{*} E \rightarrow \alpha_{*} \mathcal{H o m}_{W}(I, E) \rightarrow 0
$$

is exact for all injective $W$-modules $E$. But $\alpha_{*} \delta_{*} \delta ! E \cong \mathcal{H o m}_{X}\left(o_{Y}, \alpha_{*} E\right)$, so $\alpha_{*} \mathcal{H o m}_{W}(I, E)$ is the cokernel of the map $\mathcal{H o m}_{X}\left(o_{Y}, \alpha_{*} E\right) \rightarrow \mathcal{H}$ om $X\left(o_{X}, \alpha_{*} E\right)$. However, the exact sequence

$$
0 \rightarrow o_{X}(-Y) \rightarrow o_{X} \rightarrow o_{Y} \rightarrow 0
$$

gives rise to the exact sequence

$$
\begin{aligned}
0 \rightarrow & \mathcal{H o m}_{X}\left(o_{Y}, \alpha_{*} E\right) \rightarrow \mathcal{H o m}_{X}\left(o_{X}, \alpha_{*} E\right) \rightarrow \mathcal{H o m}_{X}\left(o_{X}(-Y), \alpha_{*} E\right) \\
& \rightarrow \mathcal{E}_{x}^{1}\left(o_{Y}, \alpha_{*} E\right) \rightarrow 0 .
\end{aligned}
$$

Therefore $\alpha_{*} \mathcal{H o m}_{W}(I, E)$ is characterized by the fact that it is the kernel in the exact sequence

$$
0 \rightarrow \alpha_{*} \mathcal{H}_{o m}(I, E) \rightarrow \mathcal{H o m}_{X}\left(o_{X}(-Y), \alpha_{*} E\right) \rightarrow \mathcal{E} x t_{X}^{1}\left(o_{Y}, \alpha_{*} E\right) \rightarrow 0 .
$$

However, if $E^{\prime}$ is an injective envelope of $\alpha_{*} E$ in $\operatorname{Mod} X$, then $E \cong \alpha^{!} E^{\prime}$, so

$$
\mathcal{E} x t_{X}^{1}\left(o_{Y}, \alpha_{*} E\right) \cong \mathcal{E} x t_{X}^{1}\left(o_{Y}, \mathcal{H}_{o m}\left(o_{W}, E^{\prime}\right)\right)=\mathcal{H} \operatorname{om}_{X}\left(\mathcal{T o r}_{1}^{X}\left(o_{Y}, o_{W}\right), E^{\prime}\right),
$$

which is zero by hypothesis. It follows that

$$
\alpha_{*} \mathcal{H o m}_{W}(I, E) \cong \mathcal{H} \operatorname{om}_{X}\left(o_{X}(-Y), \alpha_{*} E\right)
$$

and hence that

$$
\mathcal{H o m}_{W}(I,-) \cong \alpha^{\prime} \mathcal{H} \operatorname{om}_{X}\left(o_{X}(-Y),-\right) \alpha_{*}
$$

on injective $W$-modules, and hence on all $W$-modules.

However, if $F, G \in \operatorname{Lex}(X, X)$ are mutual quasi-inverses and each sends $\operatorname{Mod} W$ to itself, then $\alpha^{!} F \alpha_{*}$ and $\alpha^{!} G \alpha_{*}$ are in $\operatorname{Lex}(W, W)$ and are mutual quasi-inverses. Therefore $\mathcal{H o m}_{W}(I,-)$ is an auto-equivalence of $\operatorname{Mod} W$. 
The next two lemmas will be used later.

Lemma $8.3([26])$. If $Y$ is an effective divisor on $X$, then $\operatorname{Mod}_{Y} X$ is closed under injective envelopes in ModX.

Proof. This is proved in [26, Proposition 8.4]. In their notation, $-\otimes_{X} o_{X}(-Y)$ corresponds to the functor $G$, and $\operatorname{Mod} Y$ corresponds to $\mathcal{B}=\mathcal{D}$.

Lemma 8.4. Let $Z$ be a weakly closed subspace of a locally noetherian space $X$. Let $j: X \backslash Z \rightarrow X$ be the inclusion map. Let $\tau: \operatorname{Mod} \mathrm{M} \rightarrow \operatorname{Mod}_{Z} X \rightarrow \operatorname{Mod} X$ be the functor that takes $Z$-torsion. If $\operatorname{Mod}_{Z} X$ is closed under injective envelopes in ModX, then

1. every injective $X$-module is a direct sum of a torsion injective and a torsionfree injective;

2. for $i \geq 1$, the right-derived functors satisfy $R^{i+1} \tau M \cong R^{i} j_{*}\left(j^{*} M\right)$.

Proof. (1) Let $E$ be an injective $X$-module. Since $E$ contains a copy of the injective envelope of $\tau E$, and since that injective is torsion by hypothesis, $\tau E$ is injective. Hence $E \cong \tau E \oplus Q$, with $Q$ a torsion-free injective.

(2) Let $M \rightarrow E^{\bullet}$ be an injective resolution of $M$. For each $i$, write $I^{i}=\tau\left(E^{i}\right)$, and set $Q^{i}=E^{i} / I^{i}$. Then there is an exact sequence of complexes

$$
0 \rightarrow I^{\bullet} \rightarrow E^{\bullet} \rightarrow Q^{\bullet} \rightarrow 0
$$

which gives a long exact sequence in homology. However, $h^{i}\left(I^{\bullet}\right)=R^{i} \tau M$, and $h^{i}\left(E^{\bullet}\right)=0$ for $i \geq 1$, so $R^{i+1} \tau M \cong h^{i}\left(Q^{\bullet}\right)$ for $i \geq 1$.

Since $Q^{i}$ is torsion-free, $j_{*} Q^{i}$ is an injective $X \backslash Z$-module, and $j_{*} j^{*} Q^{i} \cong Q^{i}$. Since $j^{*}$ is exact, $j^{*} M \rightarrow j^{*} E^{\bullet}$ is an injective resolution in $\operatorname{Mod} X \backslash Z$. The complexes $j^{*} Q^{\bullet}$ and $j^{*} E^{\bullet}$ are isomorphic, so $j^{*} M \rightarrow j^{*} Q^{\bullet}$ is an injective resolution in $\operatorname{Mod} X \backslash Z$. Thus $R^{i} j_{*}\left(j^{*} M\right) \cong h^{i}\left(j_{*} j^{*} Q^{\bullet}\right) \cong h^{i}\left(Q^{\bullet}\right)$.

Proposition 8.5. Let $Y$ be an effective divisor on a locally noetherian space $X$. Let $i: Y \rightarrow X$ and $j: X \backslash Y \rightarrow X$ be the inclusions. Then

1. $j$ is an affine map;

2. $i^{!} j_{*}=i^{*} j_{*}=0$;

3. whenever $M \in \operatorname{Mod} X$ and $N \in \operatorname{Mod} X \backslash Y$,

$$
\operatorname{Ext}_{X \backslash Y}^{n}\left(j^{*} M, N\right) \cong \operatorname{Ext}_{X}^{n}\left(M, j_{*} N\right) \text {. }
$$

Proof. Let $\tau: \operatorname{Mod} X \rightarrow \operatorname{Mod}_{Y} X \rightarrow \operatorname{Mod} X$ denote the $Y$-torsion functor.

(1) By Lemma 8.4 $\left(R^{1} j_{*}\right) j^{*} \cong R^{2} \tau$. But $\tau$ is the direct limit of the functors $\mathcal{H o m}_{X}\left(o_{X} / o_{X}(-n Y),-\right)$ for $n \geq 0$, so $R^{2} \tau$ is the direct limit of the functors $\mathcal{E} x t_{X}^{2}\left(o_{X} / o_{X}(-n Y),-\right)$, each of which is zero because $o_{X}(-Y)$ is an invertible bimodule. Therefore $0=R^{2} \tau \cong\left(R^{1} j_{*}\right) j^{*}$. Since every $X \backslash Y$-module is of the form $j^{*} M$, it follows that $R^{1} j_{*}=0$, and we conclude that $j_{*}$ is exact. But $j_{*}$ commutes with direct sums (Proposition 6.8), so it has a right adjoint. Since $j^{*} j_{*} \cong \mathrm{id}_{X \backslash Y}$, $j_{*}$ is fully faithful. Hence $j$ is an affine map.

(2) The definition of $j_{*}$ ensures that $i^{1} j_{*}$ is zero. Let $N \in \operatorname{Mod} X \backslash Y$, and suppose that $i^{*} j_{*} N$ is non-zero. By (8.4), there is an exact sequence

$$
0 \rightarrow\left(j_{*} N\right)(-Y) \rightarrow j_{*} N \rightarrow i^{*} j_{*} N \rightarrow 0 .
$$

It follows from the construction of $j_{*}$ that $j_{*} N$ is not an essential extension of $\left(j_{*} N\right)(-Y)$. Hence, if $V$ denotes the image of $\left(j_{*} N\right)(-Y)$ in $j_{*} N, V \cap L=0$ for 
some non-zero submodule $L \subset j_{*} N$. But then $L$ embeds in $i^{*} j_{*} N$, so it is a $Y$ module, contradicting the fact that $\tau\left(j_{*} N\right)=0$. From this contradiction we deduce that $i^{*} j_{*} N=0$.

(3) Because $j_{*}$ is exact, the spectral sequence (2.2) for $U=X \backslash Y$ collapses, giving $\operatorname{Ext}_{X}^{n}\left(-, j_{*} N\right) \cong \operatorname{Ext}_{X \backslash Y}^{n}\left(j^{*}-, N\right)$.

Proposition 8.6. Let $Y$ be an effective divisor on $X$. If $Z$ is a weakly closed subspace of $X$ such that $Z \cap Y=\phi$, then

1. $Z$ is contained in $X \backslash Y$, and

2. $Y$ is contained in $X \backslash Z$.

Proof. Let $i: Y \rightarrow X$ and $\beta: Z \rightarrow X$ denote the inclusions. Because $Z \cap Y=0$, both $i^{!}$and $i^{*}$ vanish on all $Z$-modules. Let $M$ and $N$ be respectively a $Y$-module and a $Z$-module.

(1) Since $i^{!}$and $R^{1} i^{!}$vanish on all $Z$-modules, (8.2) gives $\operatorname{Ext}_{X}^{1}\left(i_{*} M, \beta_{*} N\right)=0$. This is precisely the criterion for $Z$ to be contained in $X \backslash Y$.

(2) Since $i^{!}$vanishes on all $Z$-modules, the only $Z$-module belonging to $\operatorname{Mod}_{Y} X$ is the zero module. Hence $\operatorname{Hom}_{X}\left(\beta_{*} N,-\right)$ is zero on $\operatorname{Mod}_{Y} X$. By Lemma 8.3 . all the terms in the minimal injective resolution of $i_{*} M$ belong to $\operatorname{Mod}_{Y} X$; hence $\operatorname{Ext}_{X}^{n}\left(\beta_{*} N, i_{*} M\right)=0$ for all $n$. Hence $Y$ is contained in $X \backslash Z$.

Corollary 8.7. Let $Y$ be an effective divisor on $X$. A closed point in $X$ is contained in either $Y$ or $X \backslash Y$ but not both, and is a closed point in whichever of these spaces contains it.

Proof. Let $p$ be a closed point of $X$ and suppose that $p$ does not lie on $Y$. By Proposition 8.6, $p$ is contained in $X \backslash Y$. As remarked in the proof of Corollary 7.3 $p$ is closed in $X \backslash Y$ and hence is a closed point of $X \backslash Y$.

It is not true, even in the commutative case, that the set of closed points in $X$ is the disjoint union of the set of closed points in $Y$ and the set of closed points in $X \backslash Y$; consider the spectrum of a discrete valuation ring. The next result gives conditions under which that is true.

Proposition 8.8. Let $X$ be a locally noetherian space. Suppose that $j: X \backslash Y \rightarrow X$ is the inclusion of the complement to an effective divisor. Let $p$ be a closed $k$ rational point of $X \backslash Y$. If $j_{*} \mathcal{O}_{p}$ is a noetherian $X$-module, then it is simple, and $j(p)$ (see Definition (5.9) is a closed k-rational point of $X$.

Proof. We will show first that if $j_{*} \mathcal{O}_{p}$ is not a simple $X$-module, then it is not noetherian. To that end, suppose that $M$ is a non-zero $X$-submodule of $j_{*} \mathcal{O}_{p}$ that is not equal to $j_{*} \mathcal{O}_{p}$.

Let $i: Y \rightarrow X$ be the inclusion and let $\tau: \operatorname{Mod} X \rightarrow \operatorname{Mod}_{Y} X$ be the $Y$-torsion functor. Since $i^{!} j_{*}=0$, we have $i^{!} M=0$ and $\tau M=0$. Hence there is an exact sequence

$$
0 \rightarrow M \rightarrow j_{*} \mathcal{O}_{p} \rightarrow R^{1} \tau M \rightarrow 0 .
$$

Since $M$ is torsion-free, $j^{*} M \neq 0$, whence $j^{*} M \cong j^{*} j_{*} \mathcal{O}_{p} \cong \mathcal{O}_{p}$. Because $i^{*} j_{*}=0$, applying (8.4) to $j_{*} \mathcal{O}_{p}$ gives $j_{*} \mathcal{O}_{p} \cong\left(j_{*} \mathcal{O}_{p}\right)(Y)$. Then applying (8.4) to the other 
two terms in (8.6) gives the other columns in the commutative diagram below:

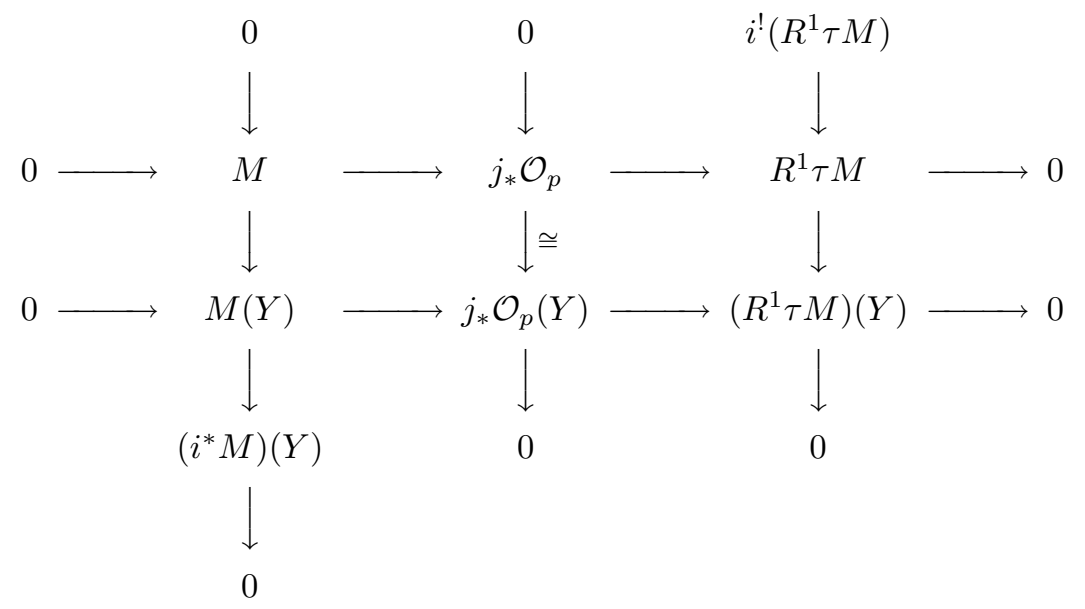

Let $M_{1}$ denote the image of $M(Y)$ in $j_{*} \mathcal{O}_{p}$. Repeating this argument produces a chain of submodules

$$
M=M_{0} \subset M_{1} \subset M_{2} \subset \cdots \subset j_{*} \mathcal{O}_{p}
$$

such that $M_{n} \cong M(n Y)$ and $M_{n} / M_{n-1} \cong\left(i^{*} M\right)(n Y)$. By the Snake Lemma, $\left(i^{*} M\right)(Y) \cong i^{!}\left(R^{1} \tau M\right)$; since $R^{1} \tau M$ is non-zero, $i^{!}\left(R^{1} \tau M\right) \neq 0$, whence $i^{*} M \neq 0$. Hence the chain of submodules is strictly increasing, thus showing that $j_{*} \mathcal{O}_{p}$ is not noetherian.

When $j_{*} \mathcal{O}_{p}$ is noetherian there can be no such $M$, so $j_{*} \mathcal{O}_{p}$ is simple. Now we show that $j_{*} \mathcal{O}_{p}$ is a tiny simple. First, $\operatorname{Hom}_{X}\left(j_{*} \mathcal{O}_{p}, j_{*} \mathcal{O}_{p}\right) \cong \operatorname{Hom}_{X \backslash Y}\left(\mathcal{O}_{p}, \mathcal{O}_{p}\right) \cong$ $k$. Let $N$ be a noetherian $X$-module. Then $j^{*} N$ is a noetherian $X \backslash Y$-module, so $\operatorname{Hom}_{X}\left(N, j_{*} \mathcal{O}_{p}\right) \cong \operatorname{Hom}_{X \backslash Y}\left(j^{*} N, \mathcal{O}_{p}\right)$ is a finite dimensional $k$-vector space. Thus $j_{*} \mathcal{O}_{p}$ is a tiny simple, so there is a closed $k$-rational point $p^{\prime} \in X$ such that $j_{*} \mathcal{O}_{p}=\mathcal{O}_{p^{\prime}}$.

Remark. The hypothesis in Proposition 8.8 does not always hold even in the commutative case: For example, let $X=\operatorname{Spec} R$ where $R$ is a discrete valuation ring. However, if $X$ is a scheme of finite type over a field $k$, then every closed $k$-rational point in the complement of an effective divisor is a closed $k$-rational point of $X$. In the non-commutative case this sort of finiteness hypothesis does not ensure that a $k$-rational closed point of $X \backslash Y$ is a closed point of $X$.

For example, consider the graded line defined by $\operatorname{Mod} \mathbb{L}^{1}=\operatorname{GrMod} k[x]$, where $\operatorname{deg} x=1$. Then $x$ cuts out an effective divisor $Y$ defined by

$$
\operatorname{Mod} Y=\operatorname{GrMod} k[x] /(x)
$$

and $\mathbb{L}^{1} \backslash Y$ is isomorphic to Spec $k\left(\operatorname{Mod}_{\mathbb{L}}^{1} \backslash Y=\right.$ Tails $\left.k[x]=\mathbb{P}_{k}^{0}\right)$. However, if $j: \mathbb{L}^{1} \backslash Y \rightarrow \mathbb{L}^{1}$ denotes the inclusion, then $j_{*} \mathcal{O}_{\mathbb{L}^{1} \backslash Y} \cong k\left[x, x^{-1}\right]$. Since $\mathbb{L}^{1} \backslash Y$ is the generic point of $\mathbb{L}^{1}$ (see [23]), it is no surprise that it is not a closed point of $\mathbb{L}^{1}$.

Corollary 8.9. Let $X$ be a locally noetherian space. Suppose that $j: X \backslash Y \rightarrow X$ is the inclusion of the complement to an effective divisor. If $j_{*} \mathcal{O}_{p}$ is a noetherian $X$-module for every closed point $p \in X \backslash Y$, then the set of closed points on $X$ is the disjoint union of the sets of closed points on $Y$ and on $X \backslash Y$. 
Proof. It is clear that a closed point on $Y$ is a closed point on $X$, so the result follows from Proposition 8.8 and Corollary 8.7

Corollary 8.9 implies that $j_{*}$ and $j^{*}$ set up a bijection between the closed points of $X$ that do not lie on $Y$, and the closed points of $X \backslash Y$.

Effective divisors on a space typically arise from regular elements in a (homogeneous) coordinate ring. For example, if $z$ is a regular element in a ring $R$ such that $z R=R z$, then $\operatorname{Mod} R /(z)$ is an effective divisor on $\operatorname{Mod} R$.

Proposition 8.10. Let $A$ be a graded ring with a central regular element $z \in A_{d}$. Set $X=$ Tails $A, H=$ Tails $A /(z)$, and let $i: H \rightarrow X$ be the inclusion. Then $H$ is an effective divisor on $X$ and $M(H) \cong M(d)$ for all $M \in \operatorname{Mod} X$. Furthermore, if $A$ is connected and generated in degree one, then $X \backslash H$ is the affine space with coordinate ring $A\left[z^{-1}\right]_{0}$.

Proof. We use the notation and terminology in [25].

Define the bimodule $o_{H}$ by $\mathcal{H}$ om $_{X}\left(o_{H},-\right)=i_{*} i^{!}$. Thus $-\otimes o_{H} \cong i_{*} i^{*}$. As usual, $o_{X}$ denotes the identity functor on $\operatorname{Mod} X$ considered as a bimodule. Define $o_{X}(-H)$ as the kernel of the map of bimodules $o_{X} \rightarrow o_{H}$ that corresponds to the counit $i_{*} i^{!} \rightarrow \mathrm{id}_{X}$. Thus $\mathcal{H}^{\circ} m_{X}\left(o_{X}(-H),-\right)$ is defined by the requirement that if $I$ is an injective $X$-module, then $\mathcal{H}^{\circ} m_{X}\left(o_{X}(-H), I\right)$ is the cokernel in the exact sequence

$$
0 \rightarrow \mathcal{H} \text { om }_{X}\left(o_{H}, I\right) \rightarrow \mathcal{H} \text { om }_{X}\left(o_{X}, I\right) \rightarrow \mathcal{H} \text { om }_{X}\left(o_{X}(-H), I\right) \rightarrow 0 .
$$

This sequence can also be written as

$$
0 \rightarrow i_{*} i^{!} I \rightarrow I \rightarrow \mathcal{H o m}_{X}\left(o_{X}(-H), I\right) \rightarrow 0 .
$$

Let $\pi: \operatorname{GrMod} A \rightarrow \operatorname{Mod} X$ be the quotient functor. There is an injective $Q$ in $\operatorname{GrMod} A$ such that $I=\pi Q$. It is easy to see that there is an exact sequence

$$
0 \rightarrow K \rightarrow Q \stackrel{\gamma}{\rightarrow} Q(d) \rightarrow 0
$$

where $\gamma(q)=q z$. Since $\pi K \cong i_{*} i^{!} I$, applying $\pi$ to this gives (8.7). Therefore $\mathcal{H}_{\text {om }}\left(o_{X}(-H), I\right) \cong I(d)$. This shows that $\mathcal{H}^{\circ} m_{X}\left(o_{X}(-H),-\right)$ is an invertible functor, and hence that $H$ is an effective divisor on $X$. The bimodule $o_{X}(H)$ is defined by requiring that $\mathcal{H}_{\text {om }}\left(o_{X}(H),-\right)$ be an inverse to $\mathcal{H}^{\circ} m_{X}\left(o_{X}(-H),-\right)$, so it follows that $I \otimes o_{X}(H) \cong I(d)$. Now, taking an injective resolution of an arbitrary $X$-module, we see that $M(H) \cong M(d)$ for all $M \in \operatorname{Mod} X$.

By definition $\operatorname{Mod} X \backslash H=\operatorname{Mod} X / \operatorname{Mod}_{H} X \cong \operatorname{GrMod} A / \mathrm{T}$, where $\mathrm{T}$ is the localizing subcategory consisting of the $A$-modules which are $z$-torsion. Thus

$$
\operatorname{GrMod} A / \mathrm{T} \cong \operatorname{GrMod} A\left[z^{-1}\right] \text {. }
$$

When $A$ is connected and generated by $A_{1}, A\left[z^{-1}\right]$ is strongly graded, meaning that 1 belongs to the product of the degree $n$ and degree $-n$ components for all $n$; this implies that $\operatorname{GrMod} A\left[z^{-1}\right]$ is equivalent to $\operatorname{Mod} A\left[z^{-1}\right]$ via the functor that takes the degree zero component of a module.

An affine scheme can be embedded in a projective scheme in such a way that it is the open complement of an ample divisor. It is well-known that a non-commutative affine space can be embedded in a non-commutative projective space in a similar way (see, for example, [4, Section 2] and [16]). We show next that closed points behave well with respect to this embedding. 
First we recall the embedding just alluded to. Let $R$ be an algebra over a field $k$. Fix a set of generators for $R$ and write $R_{n}$ for the $k$-linear span of all products of $\leq n$ of those generators. Let $t$ denote an indeterminate commuting with $R$, and set

$$
\tilde{R}:=R_{0} \oplus R_{1} t \oplus R_{2} t^{2} \oplus \cdots \subset R[t]
$$

Define

$$
U=\operatorname{Mod} R \quad \text { and } \quad X=\text { Tails } \tilde{R} .
$$

We write

$$
\pi: \operatorname{GrMod} \tilde{R} \rightarrow \operatorname{Mod} X \quad \text { and } \quad \omega: \operatorname{Mod} X \rightarrow \operatorname{GrMod} \tilde{R}
$$

for the quotient functor and its right adjoint.

The closed subspace of $X$ where $t \in \tilde{R}_{1}$ vanishes is denoted by $H$ and is called the hyperplane at infinity. Proposition 8.10 shows that $H$ is an effective divisor, because $\operatorname{Mod} H \cong \operatorname{Mod} \tilde{R} /(t)$. We also have $\operatorname{Mod} H \cong$ Tails $\operatorname{gr} R$, where $\operatorname{gr} R$ is the associated graded ring with respect to the filtration $R_{n}$. Because $R\left[t^{-1}\right]_{0} \cong R$, there is a natural map

$$
j: U \rightarrow X
$$

sending $U$ isomorphically to $X \backslash H$.

Proposition 8.11. Let $\tilde{R}$ be a finitely generated right noetherian $k$-algebra. The functors $j_{*}$ and $j^{*}$ set up a bijection between

1. the finite dimensional simple $R$-modules $V$ such that $\operatorname{End}_{R} V \cong k$ and

2. the closed $k$-rational points of $X$ that do not lie on $H$.

Proof. This will follow from Corollary 8.9 once we show that $j_{*} V$ is a noetherian $X$-module for every finite dimensional simple right $R$-module $V$. By definition, $j_{*} V \cong \pi \tilde{V}$, where $\tilde{V}$ is the graded $\tilde{R}$-module

$$
V \oplus V t \oplus V t^{2} \oplus \ldots
$$

with degree $n$ component $\tilde{V}_{n}=V t^{n}$ and action

$$
\left(v t^{n}\right) \cdot\left(r t^{i}\right):=(v r) t^{n+i}
$$

for $v \in V$ and $r \in R_{i}$. Since every component of $\tilde{V}$ has the same finite dimension, $\pi \tilde{V}$ is noetherian.

Lemma 8.12. Let $i: Y \rightarrow X$ be the inclusion of an effective divisor in a space $X$. Let $j: X \backslash Y \rightarrow X$ be the inclusion of the weakly open complement. Then $\operatorname{Ext}_{X}^{1}\left(j_{*} N, M\right)=0$ for all $N \in \operatorname{Mod} X \backslash Y$ and $M \in \operatorname{Mod} Y$.

Proof. If we apply $i^{*}$ to an exact sequence

$$
0 \rightarrow M \stackrel{\alpha}{\rightarrow} K \rightarrow j_{*} N \rightarrow 0
$$

we obtain an exact sequence

$$
\left(L_{1} i^{*}\right)\left(j_{*} N\right) \rightarrow i^{*} M \stackrel{i^{*}(\alpha)}{\longrightarrow} i^{*} K \rightarrow i^{*} j_{*} N \rightarrow 0 .
$$

The last term of this is zero by Proposition 8.5, and the first is zero because $i^{!} j_{*}=0$. Hence there is a map $\beta: i^{*} K \rightarrow i^{*} M$ such that $\beta \circ i^{*}(\alpha)=\operatorname{id}_{M}$. However, $i^{*}(\alpha)=\gamma \circ \alpha$, where $\gamma: M \rightarrow i^{*} M$ is the canonical epimorphism, so $\beta \gamma \alpha=\operatorname{id}_{M}$, which shows that the original exact sequence splits. Hence the result. 
The next result is an analogue of the result that an ample divisor on a scheme meets every subscheme of positive dimension.

Let $X$ be a locally noetherian space and $\bmod X$ its category of noetherian modules. We denote the Krull dimension, in the sense of Gabriel [6], of $M \in \bmod X$ by $\operatorname{Kdim} M$. We say that a noetherian $X$-module $M$ is $d$-critical if $\operatorname{Kdim} M=d$ but $\operatorname{Kdim} M / N<d$ for all $0 \neq N \subset M$. A noetherian module of Krull dimension $d$ has a $d$-critical quotient. We define the dimension of a weakly closed subspace $W$ of $X$ to be

$$
\operatorname{dim} W:=\max \{\operatorname{Kdim} M \mid M \in \bmod W\} .
$$

Definition 8.13. Let $X$ be a locally noetherian space endowed with a structure map $\pi: X \rightarrow$ Spec $k$. Set $\mathcal{O}_{X}=\pi^{*} k$ and $H^{q}(X,-)=\operatorname{Ext}_{X}^{q}\left(\mathcal{O}_{X},-\right)$. An effective divisor $Y$ on $X$ is ample if for every $M \in \bmod X$

- there are integers $n_{i} \leq 0$ and an epimorphism $\bigoplus \mathcal{O}_{X}\left(n_{i} Y\right) \rightarrow M$, and

- $H^{q}(X, M(n Y))=0$ if $q \neq 0$ and $n \gg 0$.

Proposition 8.14. Let $X$ be a locally noetherian space over Spec $k$, and let $Y$ be an ample effective divisor on $X$. Suppose that $\operatorname{dim}_{k} \operatorname{Ext}_{X}^{n}(M, N)<\infty$ for all $M, N \in \bmod X$. If $W$ is a weakly closed subspace of $X$ and $\operatorname{dim} W>0$, then $W \cap Y \neq \phi$.

Proof. We will assume that $W \cap Y=\phi$ and get a contradiction. Let $d=\operatorname{dim} W$ and let $M$ be a $d$-critical noetherian $W$-module. By (8.4), there is an exact sequence $0 \rightarrow i^{!} M(-Y) \rightarrow M(-Y) \rightarrow M \rightarrow i^{*} M \rightarrow 0$. Since $W \cap Y=\phi$, $i^{!} M=i^{*} M=0$, whence $M \cong M(Y)$. Hence $\operatorname{dim}_{k} H^{0}(X, M(n Y))$ is a finite number independent of $n$. By [3], $\operatorname{Mod} X$ is equivalent to Tails $B$, where $B$ is the graded algebra $\bigoplus_{n=0}^{\infty} H^{0}\left(X, \mathcal{O}_{X}(n Y)\right)$. Under this equivalence, $M \cong \pi V$, where $V$ is the graded $B$-module $\bigoplus_{n=0}^{\infty} H^{0}(X, M(n Y))$. But $\operatorname{dim} V_{n}$ is finite and independent of $n$, so $\pi V$ has finite length. This contradicts our hypothesis that $\operatorname{Kdim} M=d \geq 1$.

The next result generalizes the surprising result in [15, Lemma 6.4]. In that result, $Y$ is an effective divisor and $X \backslash Y$ is an affine space having a coordinate ring that is an infinite dimensional simple $\mathbb{C}$-algebra; such an algebra cannot have a finite dimensional simple module, so $X \backslash Y$ has no closed points.

Proposition 8.15. Let $k$ be an algebraically closed field and $X$ a locally noetherian Ext-finite space over $k$. Let $i: Y \rightarrow X$ be the inclusion of an effective divisor. Suppose that $X \backslash Y$ has no closed $k$-rational points. If $\mathcal{F}$ is a noetherian $X$-module such that $i^{*} \mathcal{F}=0$, then $\mathcal{F}=0$.

Proof. Suppose that $\mathcal{F} \neq 0$. Since $\mathcal{F}$ is noetherian, it has a simple quotient. By Corollary [5.6 that simple quotient is of the form $\mathcal{O}_{p}$ for a closed $k$-rational point $p \in X$. By the hypothesis and Corollary 8.7, $p \in Y$; this implies that $i^{*} \mathcal{F} \neq 0$.

\section{REFERENCES}

[1] M. Artin, J. Tate and M. Van den Bergh, Some algebras related to automorphisms of elliptic curves, in The Grothendieck Festschrift, Vol.1, 33-85, Birkhauser, Boston 1990. MR 92e:14002

[2] M. Artin, J. Tate and M. Van den Bergh, Modules over regular algebras of dimension 3, Invent. Math., 106 (1991) 335-388. MR 93e:16055

[3] M. Artin and J. Zhang, Non-commutative Projective Schemes, Adv. Math., 109 (1994), 228287. MR 96a:14004 
[4] M.J. Asensio, M. Van den Bergh, and F. Van Oystaeyen, A new algebraic approach to microlocalization of filtered rings, Trans. Amer. Math. Soc., 316 (1989) 537-553. MR 90c:16001

[5] G. Cauchon, Les T-anneaux, la condition (H) de Gabriel et ses conséquences, Comm. Alg., 4 (1976) 11-50. MR 52:13922

[6] P. Gabriel, Des catégories abéliennes, Bull. Soc. Math. France 90 (1962), 323-448. MR 38:1144

[7] K.R. Goodearl and R.B. Warfield, An Introduction to Noncommutative Noetherian Rings, Lond. Math. Soc. Texts, no. 16, Camb. Univ. Press, Cambridge, 1989. MR 91c:16001

[8] A. Grothendieck (with P. Berthelot and L. Illusie), Théorie des intersections et Théorème de Riemann-Roch (SGA 6), Lecture Notes in Math. 225, Springer-Verlag, Berlin/New York, 1971. MR 50:7133

[9] R. Hartshorne, Residues and Duality, Lecture Notes in Math. 20, Springer-Verlag, Berlin/New York, 1966. MR 36:5145

[10] R. Hartshorne, Algebraic Geometry, Springer-Verlag, 1977. MR 57:3116

[11] P. Jørgensen, Intersection theory on non-commutative surfaces, Trans. Amer. Math. Soc., 352 (2000) 5817-5854. MR 2001b:14005

[12] P. Jørgensen and S.P. Smith, Curves on non-commutative surfaces, in preparation.

[13] P. Jørgensen and S.P. Smith, Intersection theory for the blow-up of a non-commutative surface, in preparation.

[14] A. Joyal and R. Street, An introduction to Tannaka duality and quantum groups, pp. 413-492 in Category Theory, Proceedings, Como 1990, Eds. A. Carboni, M.C. Pedicchio, G. Rosolini, Lect. Notes in Math., vol. 1488, Springer Verlag, 1991. MR 93f:18015

[15] A. Kapustin, A. Kuznetzov, and D. Orlov, Noncommutative Instantons and Twistor Transform, Comm. Math. Phys. 221 (2001), 385-432.

[16] L. Le Bruyn and M. Van den Bergh, On quantum spaces of Lie algebras, Proc. Amer. Math. Soc., 119 (1993) 407-414. MR 93k:17021

[17] J. C. McConnell and J. C. Robson, Noncommutative Noetherian Rings, John Wiley and Sons, 1987. MR 89j:16023

[18] C. Pappacena, The injective spectrum of a non-commutative space, manuscript, February 2001, QA 0108175.

[19] N. Popescu, Abelian Categories with Applications to Rings and Modules, Academic Press (Lond. Math. Soc. Monographs), 1973. MR 49:5130

[20] A.L. Rosenberg, Non-commutative algebraic geometry and representations of quantized algebras, Vol. 330, Kluwer Academic Publishers, 1995. MR 97b:14004

[21] A.L. Rosenberg, Non-commutative schemes, Compos. Math., 112 (1998) 93-125. MR 99h:14002

[22] S.P. Smith and J. Zhang, Curves on quasi-schemes, Algebras and Representation Theory, 1 (1998) 311-351. MR 2001a:16046

[23] S.P. Smith, Integral non-commutative spaces, J. Algebra, to appear, QA 0104046.

[24] S.P. Smith, Maps between non-commutative spaces, Preprint, University of Washington, 2000.

[25] M. Van den Bergh, Blowing up on non-commutative smooth surfaces, Mem. Amer. Math. Soc., 154 (2001), No. 734.

[26] M. Van Gastel and M. Van den Bergh, Graded modules of Gelfand-Kirillov dimension one over three-dimensional Artin-Schelter regular algebras, J. Algebra, 196 (1997), 697-707. MR 99c:16020

[27] A.B. Verevkin, On a non-commutative analogue of the category of coherent sheaves on a projective scheme, Amer. Math. Soc. Transl. (2) 151 (1992) 41-53. MR 93j:14002

Department of Mathematics, Box 354350, University of Washington, Seattle, WashINGTON 98195

E-mail address: smith@math.washington.edu 